Pacific Journal of Mathematics

WEIERSTRASS POINTS WITH TWO PRESCRIBED NONGAPS 


\section{WEIERSTRASS POINTS WITH TWO PRESCRIBED NON-GAPS}

\section{COPPENS}

In this paper, we study Weierstrass points $P$ on smooth curves with two prescribed non-gaps $n$ and $s$ such that $s=e n+d$ with $0<d<n$. Let $\mathscr{M}$ be a fine moduli space of smooth curves of genus $g$ (with some extra structure) and let $\not$ : $\mathscr{X} \rightarrow \mathscr{M}$ be the associated universal family. Let $\mathscr{D}_{n, 3}=\{x \in \mathscr{X}: n$ is the first non-gap of $x$ and $\operatorname{dim}(|s x|) \geq e+1\}$. Let $Z$ be an irreducible component of $W_{n, s}$ and assume that $|s x|$ is a simple linear system on $h^{-1}(h(x))$ if $x$ is a general point on $Z$. We prove that $\operatorname{dim}(Z)=n+s+g-4-e$ and $\operatorname{dim}(|s x|)=e+1$. We give an example which shows that we cannot omit the assumption " $|s x|$ is a simple linear system". We prove that such a component $Z$ exists if and only if $e(n-1)+d \leq g \leq((n-1)(s-1)+1-(n, s)) / 2$. Finally, we derive some existence results of Weierstrass points.

Introduction. Let $C$ be a smooth, irreducible curve of genus $g \geq 1$ defined over the field $\mathbf{C}$ of the complex numbers. Let $p$ be a point on $C$. Let $n \in \mathbf{Z}_{\geq 1}$. We write $h^{0}(n p)$ instead of $\operatorname{dim}\left(H^{0}\left(C, \mathcal{O}_{C}(n p)\right)\right)$.

We say that $n$ is a gap of $p$ if $h^{0}((n-1) p)=h^{0}(n p)$. Otherwise, $n$ is a non-gap of $p$.

Using the Theorem of Riemann-Roch, one can prove that the number of gaps of $p$ is equal to $g$ and each one of them is at most equal to $2 g-1$.

We say that $\left(n_{1}, n_{2}, \ldots, n_{g}\right)$ is the gap sequence of $p$ if $1 \leq n_{1}<n_{2}<$ $\cdots<n_{g}<2 g$ and $n_{i}$ is a gap of $p$ for each $1 \leq i \leq g$. We say that $p$ is a Weierstrass point of $C$ if $n_{g} \neq g$. One can prove that $C$ has only a finite number of Weierstrass points. (For a detailed study of Weierstrass points see e.g. [14], §7d.)

Let $S \subset \mathbf{Z}_{\geq 0}$ be a sub-semigroup of $\mathbf{Z}_{\geq 0}$. Assume that there exists $c \in S$ such that $c+\mathbf{Z}_{\geq 0} \subset S$. Let $1 \leq n_{1}<n_{2}<\cdots<n_{g(S)}$ be such that $m \in Z_{\geq 0} \backslash S$ if and only if $m=n_{i}$ for some $1 \leq i \leq g(S)$. We call $\left(n_{1}, \ldots, n_{g(S)}\right)$ a gap sequence of genus $g(S)$. If there exists a smooth curve $C$ (of genus $g(S))$ and a point $p$ on $C$ such that $\left(n_{1}, n_{2}, \ldots, n_{g(S)}\right)$ is the gap sequence of $p$, then we say that $\left(n_{1}, n_{2}, \ldots, n_{g(S)}\right)$ is a Weierstrass gap sequence. It is an open problem in the theory of Weierstrass points to decide which gap sequences are Weierstrass gap sequences. There exist gap sequences which are not Weierstrass gap sequences (see [4]). 
Throughout this paper, $\mathscr{M}$ is the fine moduli space of the smooth curves of genus $g$ with a level- $\nu$-structure for some fixed $\nu \in \mathbf{Z}_{\geq 3} ; \not h$ : $\mathscr{X} \rightarrow \mathscr{M}$ is the associated smooth family of curves and $\mu: \mathscr{M} \rightarrow \mathscr{M}_{g}$ is the natural morphism to the coarse moduli space $\mathscr{M}_{g}$ of the smooth curves of genus $g$. Let $k, r \in \mathbf{Z}_{\geq 1}$ and define

$$
W_{k}^{r}=\left\{x \in \mathscr{X}: h^{0}(k x) \geq r+1\right\}
$$

(here, and throughout this paper, $h^{0}(k x)$ has to be considered with respect to the fibre $\left.h^{-1}(\not(x))\right)$. Hence $x \in W_{k}^{r}$ if and only if $x$ has at least $r$ non-gaps which are at most equal to $k$.

One has that $W_{k}^{r}$ is a closed subset of $X$ and if $Z$ is a non-empty irreducible component of it, then

$$
\operatorname{dim}(Z) \geq 3 g-2-r(g+r-k) .
$$

The proof of this statement is the same as the proof of Proposition 1 in [21].

The case $r=1$ has been studied intensively. Let us mention some results. We assume that $k \leq g-1$. In [8] and [10], it is proved that $\left.\not 1\right|_{W_{k}^{1}}$ : $W_{k}^{1} \rightarrow \mathscr{M}$ is generically injective. From the results in [1], it follows that $W_{k}^{1}$ is not empty, equidimensional of dimension $2 g-3+k$ (hence, we have equality in $(0.1))$ and $\mu\left(\not\left(W_{k}^{1}\right)\right)$ is irreducible. In particular, it follows that, for a general point $x$ on $W_{k}^{1}$, we have that

$k$ is the first non-gap of $x$.

Moreover, the gap sequence of $x$ is the hyperordinary gap sequence of genus $g$ with its first non-gap equal to $k$. This means

let $g=a(n-1)+b$ with $0 \leq b<n-1$. The non-gaps

of $x$ are the multiples of $n$ and the integers $s \geq g+a$ +1 .

This follows from [27] using the already mentioned results of [1]. It is also proved in [8] and in [11].

This paper is an attempt to prove similar results for the Weierstrass points with two prescribed non-gaps. A starting point could be the following set. Let $n, s \in \mathbf{Z}_{\geq 1}$ with $n<s, g=a(n-1)+b$ with $0 \leq b$ $<n-1$ and such that $s \leq g+a$ and $s$ is not a multiple of $n$. Let $s=e n+d$ with $0<d<n$. Consider

$$
W_{n, s}=\left\{x \in \mathscr{X}: h^{0}(n x) \geq 2 \text { and } h^{0}(s x) \geq e+2\right\} .
$$

However, this set seems too nasty to me to obtain nice (and valuable) results. I think it is better to study

$$
\stackrel{\circ}{W}_{n, s}=\left\{x \in \mathscr{X}: n \text { is the first non-gap of } x \text { and } h^{0}(s x) \geq e+2\right\} \text {. }
$$

In this paper, a satisfactory result is obtained concerning $\operatorname{dim}\left(\stackrel{\circ}{W}_{n, s}\right)$. 
At first, let me mention a "classical" work. A very general dimension formula is given in [16, p. 540-550]. However, the proof of this formula is very incomplete and, moreover, the formula does not always hold. A discussion of it can be found in [26], $\$ 4$ and a counterexample can be found in [22], p. 73. However, the key computations in $\$ 1$ of this paper are very close to the computations made in [16]. The key result in $\$ 2$, which follows from those computations is the following.

Let $Z$ be an irreducible component of $\dot{W}_{n, s}$ and let $x$ be a general point on $Z$. Assume that $|s x|$ is a simple linear system. Then

$$
\begin{aligned}
& \operatorname{dim}(Z)=n+s+g-4-e \text { and } \\
& h^{0}(s x)=e+2
\end{aligned}
$$

(in particular, $s$ is the first non-gap of $x$ which is not a multiple of $n$ ).

If $|s x|$ is not a simple linear system, then the proof does not work. Even worse, formula (0.2) does not always hold. An example of this phenomenon is given in $\$ 2$.

Hence, it is reasonable to look at

$$
\begin{array}{r}
\mathscr{W}_{n, s}=\{x \in \mathscr{X}: n \text { is the first non-gap of } x ; \\
s \text { is the first non-gap which is not a multiple of } n \\
\text { and }|s x| \text { is a simple linear system }\} .
\end{array}
$$

In $\S 3$ it is proved that $\mathscr{W}_{n, s}$ is not empty (whenever this makes sense).

Let $G_{g, n, s}=\left(n_{1}, n_{2}, \ldots, n_{g}\right)$ with $1 \leq n_{1}<n_{2}<\cdots<n_{g}$ with $n_{i} \notin$ $\left\{\alpha n+\beta s: \alpha, \beta \in \mathbf{Z}_{\geq 0}\right\}$ and if $m \in \mathbf{Z} \backslash\left\{\alpha n+\beta s: \alpha, \beta \in \mathbf{Z}_{\geq 0}\right\}$ with $m \notin\left\{n_{1}, \ldots, n_{g}\right\}$, then $m>n_{g}$ (i.e. $G_{g, n, s}$ is the gap sequence with the smallest weight having $n$ and $s$ as non-gaps).

Let $x$ be a general point on $\mathscr{W}_{n, s}$. It is natural to expect that $G_{g, n, s}$ is the Weierstrass gap sequence of $x$. I am not able to prove this statement. Therefore, this is the first of a series of questions concerning this paper.

Question 1. What is the gap sequence of a general point of $\mathscr{W}_{n, s}$ ?

In $\$ 4$, it is proved in some cases, that $G_{g, n, s}$ is a Weierstrass gap sequence, but this result is far from being complete.

Question 2. Is $\left.\not\right|_{\mathscr{W}_{n, s}}: \mathscr{W}_{n, s} \rightarrow \mathscr{M}$ generically injective?

Question 3. Is $\mu\left(\not 2\left(\mathscr{W}_{n, s}\right)\right)$ irreducible?

If Questions 2 and 3 are answered affirmatively, then Question 1 is solved if one proves that $G_{g, n, s}$ is a Weierstrass gap sequence (with the extra condition that $|s x|$ is a simple linear system). 
Question 4. If $s+1$ is not a multiple of $n$, then, does $\mathscr{W}_{n, s}$ belong to the closure of $\mathscr{W}_{n, s+1}$ ? If $s+1$ is a multiple of $n$, then, does $\mathscr{W}_{n, s}$ belong to the closure of $\mathscr{W}_{n, s+2}$ ?

This does not follow immediately from formula (0.2). Let $x$ be a general point of some irreducible component $Z$ of $\mathscr{W}_{n, s}$ (assume that $s+1$ is not a multiple of $n$ ). Let $Z^{\prime}$ be an irreducible component of $\stackrel{\circ}{W}_{n, s+1}$ containing $Z$. Then $x \in Z^{\prime}$ where the first non-gap of a general point $y$ of $Z^{\prime}$ is $n$ and its first non-gap which is not a multiple of $n$ is $s+1$. If $|(s+1) y|$ would be a simple linear system then the answer to Question 4 would be yes. If $|(s+1) y|$ is not simple then specialization to $x$ would give rise to an $(e+2)$-dimensional linear subsystem of $|(s+1) x|$ which is not simple. If $s+1$ is a gap of $x$, then $|(s+1) x|=|s x|+x$ and $|s x|$ is a simple $(e+2)$-dimensional linear system, hence we would have a contradiction. But, if $s+1$ is not a gap of $x$, then $\operatorname{dim}(|(s+1) x|)>e+2$ and, although $|(s+1) x|$ is simple, it can contain a linear subsystem which is not simple. It follows that, if the answer to Question 1 is $G_{g, n, s}$, then Question 4 is answered affirmatively.

Question 5. If $s>n+1$ and if $s$ is not a multiple of $n+1$, then does $\mathscr{W}_{n, s}$ belong to the closure of $\mathscr{W}_{n+1, s}$ (if $s$ is a multiple of $n+1$ then we take $n+1$ instead of $n$ )?

This Question can be answered affirmatively - using the dimension results of this paper - if $(e+1)(n+1)>s$. In general, the author thinks that this is a more difficult question then Question 4 (an answer to Question 1 seems not to give an answer to this one).

I thank H. C. Pinkham for the correspondence we had concerning this subject. In particular he drew by attention to the already mentioned results of [16].

\section{Models on rational surfaces and consequences.}

Construction 1. Let $g \in \mathbf{Z}_{\geq 2}$ and let $n \in \mathbf{Z}_{\geq 2}$ with $n \leq g$. Let $g=$ $a(n-1)+t$ with $0 \leq t<n-1$. Let $s \in \mathbf{Z}_{\geq 0} \backslash n \mathbf{Z}_{\geq 0}$ with $s \leq a n+t$. Let $C$ be a smooth curve of genus $g$ and assume that $p$ is a point on $C$ such that both $n$ and $s$ are non-gaps of $p$. Moreover, assume that $|s p|$ is a simple linear system on $C$. Let $l^{\prime}$ be a line in $|n p|$ such that $l^{\prime}$ has no fixed points and $n p \in l^{\prime}$. Because $|s p|$ is simple, we can find $p^{\prime} \in C \backslash\{p\}$ such that there exists $D \in|s p|$ such that, if $D^{\prime} \in l^{\prime}$ with $D^{\prime}-p^{\prime} \geq 0$, then $\inf \left(D^{\prime}+(s-n) p, D\right)=p^{\prime}$; $f D_{1}$ and $D_{2}$ are two effective divisors on $C$ then $E=\inf \left(D_{1}, D_{2}\right)$ if and only if $D_{1}-E \geq 0$ and $D_{2}-E \geq 0$ and for each $q \in C, D_{1}-E-q$ or $D_{2}-E-q$ is not an effective divisor on $C$ ]. Let $l=l^{\prime}+(s-n) p$, a line in $|s p|$, and let $g_{s}^{2}=\langle l, D\rangle$, the linear span 
of the line $l$ and the point $D$ in the projective space $|s p|$. Then $g_{s}^{2}$ is a simple linear system on $C$ which gives rise to a morphism

$$
\phi: C \rightarrow \mathbf{P}^{2} \text {. }
$$

Let $\Gamma=\Phi(C)$ and $q=\phi(p)$. Consider the surjective morphism

$$
\phi^{\prime}: C \rightarrow \Gamma \text {. }
$$

Because $l^{\prime}+(s-n) p \subset g_{s}^{2}$, one has, for each line $L$ on $\mathbf{P}^{2}$ through $q$, that

$$
\phi^{*}(L)-(s-n) p \in l^{\prime}
$$

Hence

$$
\left(\phi^{\prime}\right) *(q) \geq(s-n) p
$$

and, because $l^{\prime}$ has no fixed points, one has that

$$
\left(\phi^{\prime}\right) *(q)=(s-n) p .
$$

Moreover $\phi^{\prime}$ is birational because $g_{s}^{2}$ is simple, hence $\Gamma$ is a plane model of $C$ and $q$ is a singular point on $\Gamma$ of multiplicity $s-n$. Because $\phi^{-1}(q)=\{p\}$, the tangent cone of $\Gamma$ at $q$ is a multiple of a line $T$ on $\mathbf{P}^{2}$. Because $s p \in g_{s}^{2}$, one has that

$$
\phi^{*}(T)=s p .
$$

We are going to consider a composition of blowing-ups

$$
M \stackrel{\pi_{n}}{\rightarrow} M_{n-1} \stackrel{\pi_{n-1}}{\rightarrow} M_{n-2} \rightarrow \cdots \rightarrow M_{2} \stackrel{\pi_{2}}{\rightarrow} M_{1} \stackrel{\pi_{1}}{\rightarrow} \mathbf{P}^{2}
$$

which is determined by the singularity of $\Gamma$ at $q$. Hence, the strict transform $\tilde{\Gamma}$ of $\Gamma$ on $M$ belongs to some definite linear system $\mathbf{P}$ on $M$. Therefore, we use the following construction.

Construction 2. Let $X$ be a smooth surface, $s_{0} \in X$ and let $A$ and $B$ be reduced curves on $X$ such that $s_{0}$ is a smooth point of both $A$ and $B$ and assume that $A$ and $B$ intersect transversally at $s_{0}$. Let $D$ be a reduced curve on $X$. Assume that $D$ has a cusp in $s_{0}$ (i.e. the tangent cone of $D$ at $s_{0}$ is a multiple of a line) and assume that

$$
(D \cdot A)_{s_{0}}=(D \cdot A)=s, \quad(D \cdot B)_{s_{0}}=(D \cdot B)=n
$$

with $s \leq n$. [If $X$ is a smooth surface and $D_{1}, D_{2}$ are two divisors on $X$ and $s \in X$, then $D_{1} . D_{2}$ is the intersection cycle $\left(D_{1} . D_{2}\right)_{s}$ is the order of $D_{1} . D_{2}$ at $s$ and $\left(D_{1} . D_{2}\right)=\operatorname{deg}\left(D_{1} . D_{2}\right)$.] Let $n=a s+t$ with $0 \leq t<s$. Let $\tau_{1}: X_{1} \rightarrow X$ be the blowing-up of $X$ at $s_{0}$. Let $E_{1}$ be the exceptional divisor on $X_{1}$ such that $\tau_{1}\left(E_{1}\right)=\left\{s_{0}\right\}$. Let $D_{1}$ (resp. $B_{1}, A_{1}$ ) be the strict 
transforms of $D($ resp. $B, A)$ on $X_{1}$. We know that

$$
\begin{aligned}
& D_{1} \in\left|\left(\tau_{1}\right)^{*}(D)-s E\right|, \quad A_{1} \in\left|\left(\tau_{1}\right) *(A)-E_{1}\right| \text { and } \\
& B_{1} \in\left|\left(\tau_{1}\right) *(B)-E_{1}\right| .
\end{aligned}
$$

Let $\left\{s_{1}\right\}=D_{1} \cap E_{1}$. We know that

$$
\begin{aligned}
& \left(D_{1} \cdot E_{1}\right)_{s_{1}}=\left(D_{1} \cdot E_{1}\right)=s_{1}, \quad\left(D_{1} \cdot A_{1}\right)_{s_{1}}=\left(D_{1} \cdot A_{1}\right)=0, \\
& \left(D_{1} \cdot B_{1}\right)_{s_{1}}=\left(D_{1} \cdot B_{1}\right)=(a-1) s+t .
\end{aligned}
$$

Continuing this construction, we obtain a composition of blowing-ups

$$
X_{a} \stackrel{\tau_{a}}{\rightarrow} X_{a-1} \stackrel{\tau_{u-1}}{\rightarrow} X_{a-2} \rightarrow \cdots \rightarrow X_{2} \stackrel{\tau_{2}}{\rightarrow} X_{1} \stackrel{\tau_{1}}{\rightarrow} X
$$

such that the following properties hold.

For $a>i>0$, let $s_{l}$ be the point on $X_{l}$ blowing-up by $\tau_{t}$. For $a+1>i>0$, let $E_{l}$ be the exceptional divisor on $X_{l}$ such that $\tau_{i}\left(E_{i}\right)=$ $\left\{s_{l-1}\right\}$. For $a+1>i>0$, let $D_{i}$ be the strict transform of $D$ on $X_{i}$. For $a>i>0$, one has that $D_{i} \cap E_{i}=\left\{s_{i}\right\}$.

Let $B_{a}$ be the strict transform of $B$ on $X_{a}$. Let $D_{a} \cap B_{a}=\left\{s_{a}\right\}$. One has that

$$
\left(D_{a} \cdot E_{a}\right)_{s_{a}}=\left(D_{a} \cdot E_{a}\right)=s, \quad\left(D_{a} \cdot B_{a}\right)_{s_{a}}=\left(D_{a} \cdot B_{a}\right)=t .
$$

Hence, if $t \neq 0$, then $s_{a}$ is a cusp of $D_{a}$ of multiplicity $t$.

Construction 3. (This is a continuation of Construction 1.) Define $m \in \mathbf{Z}_{\geq 1}$ and $\left\{a_{1}, \ldots, a_{m-1}, s_{1}, \ldots, s_{m}, n_{1}, \ldots, n_{m-1}\right\} \subset \mathbf{Z}$ as follows $s_{1}=s-n$ and $n_{1}=s$; for $0<i<m$, one has that $n_{i}=a_{i} s_{l}+s_{i+1}$ with $0 \leq s_{i+1}<s_{i}$; for $1<i<m$, one has $n_{i}=s_{i-1} ; s_{m} \in\{0,1\}$ and $s_{m-1} \notin$ $\{0,1\}$. Let $M_{0}=\mathbf{P}^{2}$. For $1 \leq i \leq a_{1}+\cdots+a_{m-1}=\varepsilon$, let $\pi_{i}: M_{i} \rightarrow M_{i-1}$ be the blowing-up of $M_{1-1}$ such that the following properties hold.

Let $E_{l}$ be the exceptional divisor on $M_{i}$ blowing-down by $\pi_{i}$ and let $\Gamma_{l}$ be the strict transform of $\Gamma$ on $M_{i}$. One has that $E_{i} \cap \Gamma_{i}=\left\{q_{i}\right\}$ and $\left(\pi_{1} \circ \cdots \circ \pi_{i}\right)\left(q_{l}\right)=q$. (This is a repetition of $m-1$ times Construction 2.)

Let $\mu_{l}=\pi_{1} \circ \cdots \circ \pi_{i}$. One finds that

$$
\Gamma_{\varepsilon} \in\left|\left(\mu_{\varepsilon}\right) *(s L)-\sum\left(\left(\mu_{\varepsilon-1}\right) *\left(c_{l} E_{l}\right): 0<i<\varepsilon\right)-s_{m-1} E_{\varepsilon}\right|
$$

with, for $1+a_{1}+\cdots+a_{k} \leq i \leq a_{1}+\cdots+a_{k+1}$, one has that $c_{l}=s_{k+1}$ (here, $L$ is an arbitrary line on $\mathbf{P}^{2}$ ).

Case A. Assume that $s_{m}=1$ (i.e. $n$ and $s$ are relatively prime).

Let $E^{\prime}$ be the strict transform of $E_{\varepsilon-a_{m-1}}$ on $M_{\varepsilon}$. One has that $E_{\varepsilon} \cap E^{\prime}=\Gamma_{\varepsilon} \cap E^{\prime}=\left\{q_{\varepsilon}\right\}$ and moreover $\left(E^{\prime} . \Gamma_{\varepsilon}\right)_{q_{\varepsilon}}=\left(E^{\prime} . \Gamma_{\varepsilon}\right)=1$ and $\left(E_{\varepsilon} \cdot \Gamma_{\varepsilon}\right)_{q_{\varepsilon}}=\left(E_{\varepsilon} \cdot \Gamma_{\varepsilon}\right)=s_{m-1}$. 
Consider the composition of blowing-ups

$$
M=M_{\varepsilon+s_{m-1}} \stackrel{\pi_{\varepsilon+s_{m-1}}}{\rightarrow} M_{\varepsilon+s_{m-1}-1} \rightarrow \cdots \rightarrow M_{\varepsilon+1} \stackrel{\pi_{\varepsilon+1}}{\rightarrow} M_{\varepsilon}
$$

defined as follows.

The morphism $\pi_{\varepsilon+1}$ is the blowing-up of $M_{\varepsilon}$ at $q_{\varepsilon}$. For $\varepsilon+s_{m-1}>i$ $>\varepsilon$, let $q_{i}$ be the point on $M_{i}$ blowing-up by $\pi_{i+1}$. Then

$$
\left(\pi_{i} \circ \cdots \circ \pi_{\varepsilon+1}\right)\left(q_{i}\right)=\left\{q_{\varepsilon}\right\} .
$$

For $\varepsilon+s_{m-1} \geq i>\varepsilon$, let $E_{i}$ be the exceptional divisor on $M_{i}$ blowingdown by $\pi_{i}$ and let $\left(E_{\varepsilon}\right)_{i}$ be the strict transform of $E_{\varepsilon}$ on $M_{i}$. Then, for $\varepsilon+s_{m-1}>i>\varepsilon$, one has that $E_{i} \cap\left(E_{\varepsilon}\right)_{i}=\left\{q_{i}\right\}$.

Let $\tilde{\Gamma}$ be the strict transform of $\Gamma$ on $M$. For $0 \leq j \leq s_{m-1}$, let $\gamma_{j}=\pi_{\varepsilon+j+1} \circ \cdots \circ \pi_{\varepsilon+s_{m-1}}$. Then

$$
\tilde{\Gamma} \in\left|\left(\gamma_{0}\right) *\left(\Gamma_{\varepsilon}\right)-\sum\left(\left(\gamma_{j-1}\right) *\left(E_{\varepsilon+j-1}\right): 2 \leq j \leq s_{m-1}\right)-E_{\varepsilon+s_{m-1}}\right| .
$$

Case B. Assume that $s_{m}=0$ (i.e. $n$ and $s$ are not relatively prime).

Let $E^{\prime}$ be as before and let $\left\{q_{\varepsilon}\right\}=E_{\varepsilon} \cap \Gamma_{\varepsilon}$. One has that $E^{\prime} \cap \Gamma_{\varepsilon}=\varnothing$ and $\left(E_{\varepsilon} \cdot \Gamma_{\varepsilon}\right)_{q_{\varepsilon}}=s_{m-1}$. Assume that $q_{\varepsilon}$ is a point of multiplicity $\kappa$ on $\Gamma_{\varepsilon}$.

Case B1. Assume that $\kappa=s_{m-1}$. Let $\pi_{\varepsilon+1}: M_{\varepsilon+1} \rightarrow M_{\varepsilon}$ be the blowing-up of $M_{\varepsilon}$ at $q_{\varepsilon}$. Let $\Gamma_{\varepsilon+1}$ be the strict transform of $\Gamma_{\varepsilon}$ on $M_{\varepsilon+1}$. Let $E_{\varepsilon+1}$ be the exceptional divisor on $M_{\varepsilon+1}$ blowing-down by $\pi_{\varepsilon+1}$. One has that

$$
\Gamma_{\varepsilon+1} \in\left|\left(\pi_{\varepsilon+1}\right) *\left(\Gamma_{\varepsilon}\right)-s_{m-1} E_{\varepsilon+1}\right| .
$$

Let $\Gamma_{\varepsilon+1} \cap E_{\varepsilon+1}=\left\{q_{\varepsilon+1}\right\}$. One has that

$$
\left(\Gamma_{\varepsilon+1} \cdot E_{\varepsilon+1}\right)_{q_{\varepsilon+1}}=\left(\Gamma_{\varepsilon+1} \cdot E_{\varepsilon+1}\right)=s_{m-1} .
$$

Case B1A. $q_{\varepsilon+1}$ is a smooth point on $\Gamma_{\varepsilon+1}$.

Let $\pi_{\varepsilon+2}: M_{\varepsilon+2} \rightarrow M_{\varepsilon+1}$ be the blowing-up of $M_{\varepsilon+1}$ at $q_{\varepsilon+1}$ and let $E_{\varepsilon+2}$ be the exceptional divisor on $M_{\varepsilon+2}$ blowing-down by $\pi_{\varepsilon+2}$. Let $\Gamma_{\varepsilon+2}$ (resp. $\left(E_{\varepsilon+1}\right)_{\varepsilon+2}$ be the strict transform of $\Gamma_{\varepsilon+1}$ (resp. $E_{\varepsilon+1}$ ) on $M_{\varepsilon+2}$. One has that

$$
\Gamma_{\varepsilon+2} \in\left|\left(\pi_{\varepsilon+2}\right) *\left(\Gamma_{\varepsilon+1}\right)-E_{\varepsilon+2}\right| .
$$

Because $s_{m-1}>1$, one has that

$$
\Gamma_{\varepsilon+2} \cap E_{\varepsilon+2}=\Gamma_{\varepsilon+2} \cap\left(E_{\varepsilon+1}\right)_{\varepsilon+2}=\left\{q_{\varepsilon+2}\right\} \text {. }
$$


Let $\pi_{\varepsilon+3}: M \rightarrow M_{\varepsilon+2}$ be the blowing-up of $M_{\varepsilon+2}$ at $q_{\varepsilon+2}$. Let $E_{\varepsilon+3}$ be the exceptional divisor on $M$ blowing-down by $\pi_{\varepsilon+3}$ and let $\tilde{\Gamma}$ be the strict transform of $\Gamma_{\varepsilon+2}$ on $M$. One has that

$$
\tilde{\Gamma} \in\left|\left(\pi_{\varepsilon+3}\right) *\left(\Gamma_{\varepsilon+2}\right)-\left(\Gamma_{\varepsilon+3}\right)\right| \text {. }
$$

Case B1B. $q_{\varepsilon+1}$ is not a smooth point on $\Gamma_{\varepsilon+1}$.

Let $\pi_{\varepsilon+2}: M \rightarrow M_{\varepsilon+1}$ be the blowing-up of $M_{\varepsilon+1}$ at $q_{\varepsilon+1}$. Let $E_{\varepsilon+2}$ be the exceptional divisor on $M$ blowing-down by $\pi_{\varepsilon+2}$ and let $\tilde{\Gamma}$ be the strict transform of $\Gamma_{\varepsilon+1}$ on $M$. One has that

$$
\tilde{\Gamma} \in\left|\left(\pi_{\varepsilon+2}\right) *\left(\Gamma_{\varepsilon+1}\right)-\rho E_{\varepsilon+2}\right|
$$

with $\rho \in \mathbf{Z}_{>1}$.

Case B2. $\kappa<s_{m-1}$.

Let $s_{m-1}=\kappa \alpha+\beta$ with $0 \leq \beta<\kappa$. Let

$$
M_{\varepsilon+\alpha} \stackrel{\pi_{\varepsilon+\alpha}}{\rightarrow} M_{\varepsilon+\alpha-1} \rightarrow \cdots \rightarrow M_{\varepsilon+1} \stackrel{\pi_{\varepsilon+1}}{\rightarrow} M_{\varepsilon}
$$

be the composition of blowing-ups similar to Construction 2. Let $\Gamma_{\varepsilon+\alpha}$ be the strict transform of $\Gamma_{\varepsilon}$ on $M_{\varepsilon+\alpha}$. For $1 \leq i \leq \alpha$, let $E_{\varepsilon+i}$ be the exceptional divisor on $M_{\varepsilon+i}$ blowing-down by $\pi_{\varepsilon+i}$ and for $0 \leq i<\alpha$, let $\gamma_{l}=\pi_{\varepsilon+1+1} \circ \cdots \circ \pi_{\varepsilon+\alpha}$. One has that

$$
\Gamma_{\varepsilon+\alpha} \in\left|\left(\gamma_{0}\right) *\left(\Gamma_{\varepsilon}\right)-\sum\left(\kappa\left(\gamma_{i}\right) *\left(E_{\varepsilon+i}\right): 0<i<\alpha\right)-\kappa E_{\varepsilon+\alpha}\right| .
$$

Case B2A. $\kappa=1$.

Then, let $M=M_{\varepsilon+\alpha}$ and let $\tilde{\Gamma}=\Gamma_{\varepsilon+\alpha}$.

Case B2B. $\kappa>1$ and $\beta=0$.

Let $E^{\prime \prime}$ be the strict transform of $E_{\varepsilon}$ on $M_{\varepsilon+\alpha}$. One has that

$$
\begin{gathered}
E^{\prime \prime} \cap \Gamma_{\varepsilon+\alpha}=\varnothing \quad \text { and } \Gamma_{\varepsilon+\alpha} \cap E_{\varepsilon+\alpha}=\left\{q_{\varepsilon+\alpha}\right\} \quad \text { with } \\
\left(\Gamma_{\alpha+\varepsilon} \cdot E_{\alpha+\varepsilon}\right)_{q_{\alpha+\varepsilon}}=\left(\Gamma_{\alpha+\varepsilon} . E_{\alpha+\varepsilon}\right)=\kappa .
\end{gathered}
$$

Case B2B1. Assume that $q_{\varepsilon+\alpha}$ is a singular point on $\Gamma_{\alpha+\varepsilon}$.

Let $\pi_{\alpha+\varepsilon+1}: M \rightarrow M_{\alpha+\varepsilon}$ be the blowing-up of $M_{\alpha+\varepsilon}$ at $q_{\alpha+\varepsilon}$. Let $E_{\alpha+\varepsilon+1}$ be the exceptional divisor on $M$ blowing-down by $\pi_{\alpha+\varepsilon+1}$. Let $\tilde{\Gamma}$ be the strict transform of $\Gamma_{\alpha+\varepsilon}$ on $M$. One has that

$$
\tilde{\Gamma} \in\left|\left(\pi_{\alpha+\varepsilon+1}\right) *\left(\Gamma_{\alpha+\varepsilon}\right)-\rho E_{\alpha+\varepsilon+1}\right|
$$

with $\rho \in \mathbf{Z}_{>1}$.

Case B2B2. Assume that $q_{\alpha+\varepsilon}$ is not a singular point on $\Gamma_{\alpha+\varepsilon}$. 
Let $\pi_{\alpha+\varepsilon+1}: M_{\alpha+\varepsilon+1} \rightarrow M_{\alpha+\varepsilon}$ be the blowing-up of $M_{\alpha+\varepsilon}$ at $q_{\alpha+\varepsilon}$. Let $E_{\alpha+\varepsilon+1}$ be the exceptional divisor on $M_{\alpha+\varepsilon+1}$ blowing-down by $\pi_{\alpha+\varepsilon+1}$. Let $\left(E_{\alpha+\varepsilon}\right)^{\prime}$ be the strict transform of $E_{\alpha+\varepsilon}$ on $M_{\alpha+\varepsilon+1}$. Let $\Gamma_{\alpha+\varepsilon+1}$ be the strict transform of $\Gamma_{\alpha+\varepsilon}$ on $M_{\alpha+\varepsilon+1}$. One has that, because $\kappa>1$,

$$
\begin{aligned}
\left(E_{\alpha+\varepsilon}\right)^{\prime} \cap E_{\alpha+\varepsilon+1} & =E_{\alpha+\varepsilon+1} \cap \Gamma_{\alpha+\varepsilon+1} \\
& =\left(E_{\alpha+\varepsilon}\right)^{\prime} \cap \Gamma_{\alpha+\varepsilon+1}=\left\{q_{\alpha+\varepsilon+1}\right\} .
\end{aligned}
$$

Let $\pi_{\alpha+\varepsilon+2}: M \rightarrow M_{\alpha+\varepsilon+1}$ be the blowing-up of $M_{\alpha+\varepsilon+1}$ at $q_{\alpha+\varepsilon+1}$. Let $E_{\alpha+\varepsilon+2}$ be the exceptional divisor on $M$ blowing-down by $\pi_{\alpha+\varepsilon+2}$. Let $\tilde{\Gamma}$ be the strict transform of $\Gamma_{\alpha+\varepsilon+1}$ on $M$. One has that

$$
\tilde{\Gamma} \in\left|\left(\pi_{\alpha+\varepsilon+1} \circ \pi_{\alpha+\varepsilon+2}\right) *\left(\Gamma_{\alpha+\varepsilon}\right)-\left(\pi_{\alpha+\varepsilon+2}\right) *\left(E_{\alpha+\varepsilon+1}\right)-E_{\alpha+\varepsilon+2}\right| .
$$

Case B2C. $\beta \geq 1$.

Let $E^{\prime}$ be the strict transform of $E_{\varepsilon}$ on $M_{\alpha+\varepsilon}$. One has that

$$
\begin{aligned}
& E_{\alpha+\varepsilon} \cap \Gamma_{\alpha+\varepsilon}=E^{\prime} \cap E_{\alpha+\varepsilon}=\left\{q_{\alpha+\varepsilon}\right\} \text { and } \\
& \left(E_{\alpha+\varepsilon} \cdot \Gamma_{\alpha+\varepsilon}\right)_{q_{\alpha+\varepsilon}}=\left(E_{\alpha+\varepsilon} \cdot \Gamma_{\alpha+\varepsilon}\right)=\kappa, \\
& \left(E^{\prime} \cdot \Gamma_{\alpha+\varepsilon}\right)_{q_{\varepsilon+\alpha}}=\left(E^{\prime} \cdot \Gamma_{\alpha+\varepsilon}\right)=\beta,
\end{aligned}
$$

hence $q_{\alpha+\varepsilon}$ is a cusp of multiplicity $\beta$ on $\Gamma_{\alpha+\varepsilon}$. Let $\pi_{\alpha+\varepsilon+1}: M_{\alpha+\varepsilon+1} \rightarrow$ $M_{\alpha+\varepsilon}$ be the blowing-up of $M_{\alpha+\varepsilon}$ at $q_{\alpha+\varepsilon}$. Let $\Gamma_{\alpha+\varepsilon+1}$ be the strict transform of $\Gamma_{\alpha+\varepsilon}$ on $M_{\alpha+\varepsilon+1}$. Let $E_{\alpha+\varepsilon+1}$ be the exceptional divisor on $M$ blowing-down by $\pi_{\alpha+\varepsilon+1}$. Then

$$
\Gamma_{\alpha+\varepsilon+1} \in\left|\left(\pi_{\alpha+\varepsilon+1}\right) *\left(\Gamma_{\alpha+\varepsilon}\right)-\beta E_{\alpha+\varepsilon+1}\right| .
$$

Case B2C1. $\kappa-\beta<\beta$.

Let $\left(E_{\alpha+\varepsilon}\right)_{\alpha+\varepsilon+1}$ be the strict transform of $E_{\alpha+\varepsilon}$ on $M_{\alpha+\varepsilon+1}$. Because $\kappa-\beta \geq 1$, one has that

$$
\left(E_{\alpha+\varepsilon}\right)_{\alpha+\varepsilon+1} \cap E_{\alpha+\varepsilon+1}=\Gamma_{\alpha+\varepsilon+1} \cap E_{\alpha+\varepsilon+1}=\left\{q_{\alpha+\varepsilon+1}\right\} .
$$

Let $\pi_{\alpha+\varepsilon+2}: M \rightarrow M_{\alpha+\varepsilon+1}$ be the blowing-up of $M_{\alpha+\varepsilon+1}$ at $q_{\alpha+\varepsilon+1}$. Let $E_{\alpha+\varepsilon+2}$ be the exceptional divisor on $M$ blowing-down by $\pi_{\alpha+\varepsilon+2}$. Let $\tilde{\Gamma}$ be the strict transform of $\Gamma_{\alpha+\varepsilon+1}$ on $M$. One has that

$$
\tilde{\Gamma} \in\left|\left(\pi_{\alpha+\varepsilon+2}\right) *\left(\Gamma_{\alpha+\varepsilon+1}\right)-(\kappa-\beta) E_{\alpha+\varepsilon+2}\right| .
$$

Case B2C2. $\beta \leq \kappa-\beta$.

Let $\left(E_{\alpha+\varepsilon}\right)_{\alpha+\varepsilon+1}$ be the strict transform of $E_{\alpha+\varepsilon}$ on $M_{\alpha+\varepsilon+1}$. Because $\kappa>\beta$, one has that

$$
\left(E_{\alpha+\varepsilon}\right)_{\alpha+\varepsilon+1} \cap E_{\alpha+\varepsilon+1}=\Gamma_{\alpha+\varepsilon+1} \cap E_{\alpha+\varepsilon+1}=\left\{q_{\alpha+\varepsilon+1}\right\} .
$$


Let $\pi_{\alpha+\varepsilon+2}: M \rightarrow M_{\alpha+\varepsilon+1}$ be the blowing-up of $M_{\alpha+\varepsilon+1}$ at $q_{\alpha+\varepsilon+1}$. Let $E_{\alpha+\varepsilon+2}$ be the exceptional divisor on $M$ blowing-down by $\pi_{\alpha+\varepsilon+2}$. Let $\tilde{\Gamma}$ be the strict transform of $\Gamma_{\alpha+\varepsilon+1}$ on $M$. One has that

$$
\tilde{\Gamma} \in\left|\left(\pi_{\alpha+\varepsilon+2}\right) *\left(\Gamma_{\alpha+\varepsilon+1}\right)-\beta E_{\alpha+\varepsilon+2}\right| .
$$

RemarK 4. In Construction 3, the points $q_{1}$ which are blown-up, are completely determined by the exceptional divisors and their strict transforms, except in the following cases:

$$
\begin{aligned}
& q_{\varepsilon} \text { in Case B, } \\
& q_{\varepsilon+1} \text { in Case B1, } \\
& q_{\varepsilon+\alpha} \text { in Case B2B. }
\end{aligned}
$$

THEOREM 5. Let $(n, s)$ be the greatest common divisor of $n$ and $s$. If $g>((n-1)(s-1)+1-(n, s)) / 2$, then $\mathscr{W}_{n, s}=\varnothing$.

Proof. Let $C, M_{\varepsilon}$ and $\Gamma_{\varepsilon}$ be as in Construction 3. We have that $g(C) \leq p_{a}\left(\Gamma_{\varepsilon}\right)$ where $g(C)$ (resp. $p_{a}\left(\Gamma_{\varepsilon}\right)$ ) is the genus (resp. arithmetic genus) of $C$ (resp. $\Gamma_{\varepsilon}$ ). Because $\Gamma_{\varepsilon}$ is an irreducible curve on a rational surface $M$, we have that

$$
p_{a}\left(\Gamma_{\varepsilon}\right)=\left(\left(K_{M_{\varepsilon}}+\Gamma_{\varepsilon} \cdot \Gamma_{\varepsilon}\right)+2\right) / 2,
$$

where $K_{M_{\varepsilon}}$ is some canonical divisor on $M_{\varepsilon}$ (adjunction formula). But

$$
\left|K_{M_{\varepsilon}}\right|=\left|\left(\mu_{\varepsilon}\right) *(-3 L)+\left(\sum\left(\mu_{\varepsilon-i}\right) *\left(E_{i}\right): 0<i<\varepsilon\right)+E_{\varepsilon}\right|
$$

hence

$$
\begin{array}{r}
\left|K_{M_{\varepsilon}}+\Gamma_{\varepsilon}\right|=\mid\left(\mu_{\varepsilon}\right) *((s-3) L)-\left(\sum\left(\mu_{\varepsilon-1}\right) *\left(\left(c_{i}-1\right) E_{i}\right): 0<i<\varepsilon\right) \\
-\left(s_{m-1}-1\right) E_{\varepsilon} \mid
\end{array}
$$

and

$$
\begin{aligned}
&\left(K_{M_{\varepsilon}}+\Gamma_{\varepsilon} \cdot \Gamma_{\varepsilon}\right)=s(s-3)-\sum\left(c_{i}\left(c_{i}-1\right): 0<i<\varepsilon\right)-s_{m-1}\left(s_{m-1}-1\right) \\
&= s(s-3)-\sum\left(a_{i} s_{l}\left(s_{i}-1\right): 1 \leq i \leq m-1\right) \\
&=\left(s^{2}-\sum\left(a_{l} s_{l}^{2}: 1 \leq i \leq m-1\right)\right)-\left(3 s-\sum\left(a_{i} s_{i}: 1 \leq i \leq m-1\right)\right) \\
&=\left(s^{2}-\sum\left(s_{i}\left(n_{i}-s_{i+1}\right): 1 \leq i \leq m-1\right)\right) \\
&-\left(3 s-\sum\left(n_{i}-s_{i+1}: 1 \leq i \leq m-1\right)\right)
\end{aligned}
$$




$$
\begin{aligned}
= & \left(s^{2}-\sum\left(n_{i+1}\left(n_{i}-n_{i+2}\right): 1 \leq i \leq m-3\right)-n_{m-1}\left(n_{m-2}-s_{m-1}\right)\right. \\
& -\left(3 s-\sum\left(n_{i}-n_{t+2}: 1 \leq i \leq m-3\right)\right. \\
& -\left(n_{m-1}\left(n_{m-1}-s_{m}\right)\right) \\
= & s^{2}-n_{1} n_{2}+s_{m-1} s_{m}-\left(3 s-n_{1}-n_{2}+s_{m-1}+s_{m}\right) \\
= & \left.s n-s-n+1+\left(s_{m-1}-s_{m}\right)\right)
\end{aligned}
$$

Let $\tau=s_{m-1} s_{m}-s_{m-1}-s_{m}-1$. Hence

$$
\left(K_{M_{\varepsilon}}+\Gamma_{\varepsilon} \cdot \Gamma_{\varepsilon}\right)=(s-1)(n-1)+\tau \text {. }
$$

If $s_{m}=1$, then $\tau=-1-(s, n)=-2$. If $s_{m}=0$, then $\tau=-1-(s, n)=$ $-1-s_{m-1}$. Hence, in both cases, we obtain that

$$
\left(K_{M_{\varepsilon}}+\Gamma_{\varepsilon}, \Gamma_{\varepsilon}\right)=(s-1)(n-1)-(s, n)-1 .
$$

Hence, $p_{a}\left(\Gamma_{\varepsilon}\right)=((s-1)(n-1)+1-(s, n)) / 2$, which completes the proof of this Theorem.

2. On a formula of Hensel and Landsberg. In [16, S. 547-548], a certain statement is claimed which could be interpreted as follows.

Statement 6 (incorrect). Let $C$ be a smooth curve of genus $g$ and let $p$ be a Weierstrass point on $C$ whose first non-gap is equal to $n$ and whose first non-gap relatively prime to $n$ is $n+r$. Let $G(p)$ be the gap sequence of $p$ and let

$W_{G(p)}=\left\{x \in \mathscr{X}:\right.$ the gap sequence of $x$ on $\mathfrak{h}^{-1}(\not p(x))$ is equal to $\left.G(p)\right\}$.

Let $c \in \mathscr{X}$ such that $\not^{-1}(\not 2(c)) \cong C$ and $c$ corresponds to $p$ under this isomorphism. Let $A$ be an irreducible component of $G(p)$ containing $c$. Then

$$
\operatorname{dim}(A)=g-3+2 n+r-h,
$$

where $h+1=h^{0}((n+r) p)$.

A counterexample to this statement can be found in [22, p. 73]. Namely, if $g=4$, then $W_{(1,2,3,7)}$ is equidimensional of dimension 7 while the above formula gives 8 . Moreover, it is proved in [25], Theorem (14.5) that $W_{(1,2,3,7)}$ is not empty.

However, following the arguments used by Hensel and Landsberg, it seems to me that they tried to obtain the following result. 
Statement 7 (incorrect). Let $(n, r, h) \in \mathbf{Z}^{3}$ and let $W_{n, r, h}=\{x \in \mathscr{X}$ : the first non-gap of $x$ is equal to $n$, the first non-gap of $x$ relatively prime to $n$ is equal to $n+r$ and $\left.h+1=h^{0}((n+r) p)\right\}$.

If $W_{n, r, h} \neq \varnothing$ then $W_{n, r, h}$ is equidimensional of dimension $g-3+$ $2 n+r-h$.

Lax's example mentioned before does not give a counterexample to this statement. Indeed, $W_{(1,2,3,7)} \subset W_{4,1,2}$, but $W_{(1,2,3,7)}$ is contained in the closure of $W_{(1,2,3,6)}$ and it is proved in [22] that $W_{(1,2,3,6)}$ is equidimensional of dimension 8 which is in agreement with Statement 7. Nevertheless, the arguments used by Hensel and Landsberg are far from rigorous and it seems to me that they can be modified only to obtain the following statement.

Statement 8 (correct). Let $(n, r) \in \mathbf{Z}^{2}$ and let $G_{n, r}=\{x \in \mathscr{X}: n$ is the first non-gap of $x$ and $n+r$ is the first non-gap of $x$ relatively prime to $n$ and $h^{0}((n+r) x)=h+2$ where $n+r=h n+\varepsilon$ with $\left.0 \leq \varepsilon<n\right\}$.

If $G_{n, r} \neq \varnothing$, then $G_{n, r}$ is equidimensional of dimension $g-4+2 n$ $+r-h$. This statement follows from Theorem 11 .

Statement 7 is indeed false, as it can be seen from the following example.

EXAMPLE 9. Let $g=8$ and consider $W_{4,9,6}$. Let $x \in W_{4,9,6}$. If 6 is a gap of $x$, then $1,2,3,5,6,7,9,11$ are gaps of $x$ and therefore $h^{0}(13 x) \leq 6$, a contradiction to $h^{0}(13 x)=7$. Thus, 6 is not a gap of $x$. Hence, the only possible gaps of $x$ are $1,2,3,5,7,9,11,15$. Because $g=8$, we obtain that

$$
W_{4,9,6}=W_{(1,2,3,5,7,9,11,15)} \text {. }
$$

This is not empty because of [19, Theorem 4.14]. But, from [19, Proposition 2.9], it follows that $h^{-1}(\not(x))$ is elliptic-hyperelliptic (i.e. a double covering of an elliptic curve). Hence $\not\left(W_{4,9,6}\right)$ is contained in the so-called elliptic-hyperelliptic locus. Hence, because of [20, Satz I],

$$
\operatorname{dim}\left(\not h\left(W_{4,9,6}\right)\right) \leq 14 \text {. }
$$

But $\operatorname{dim}\left(\not\left(W_{4,9,6}\right)\right)=\operatorname{dim}\left(W_{4,9,6}\right)$ [a curve has only a finite number of Weierstrass points] and Statement 7 would imply that

$$
\operatorname{dim}\left(W_{4,9,6}\right)=8-3+8+9-6=16,
$$

hence a contradiction.

The following lemma is very important for the sequel of this paper.

Lemma 10. Let $g \in \mathbf{Z}_{\geq 2}$ and let $n \in \mathbf{Z}_{\geq 2}$ with $n \leq g$. Let $g=$ $a(n-1)+t$ with $0 \leq t<n-1$. Let $s \in \mathbf{Z}_{\geq n} \backslash n Z_{\geq 0}$ with $s \leq$ an $+t$. 
Let $s=e n+d$ with $0<d<n$. Assume there exists $x \in \mathscr{X}$ with

$$
h^{0}(n x)=2 \text { and } h^{0}(s x)=e+1+\varepsilon
$$

where $\varepsilon \in \mathbf{Z}_{>1}$. Let $Z$ be an irreducible component of

$$
\left\{z \in \mathscr{X}: h^{0}(n z) \geq 2 \text { and } h^{0}(s x) \geq e+2\right\}
$$

containing $x$, then

$$
\operatorname{dim}(Z) \geq n+s+g-4-e .
$$

Proof. Consider the family of smooth curves of genus $g$

$$
\not{p}: \mathscr{X} \times_{\mathscr{M}} \mathscr{X} \rightarrow \mathscr{X} .
$$

This family has a natural section $s$ such that for $z \in \mathscr{X}$ the point $s(z)$ on $\not^{-1}(z)$ corresponds to $z$ on $\not h^{-1}(\not h(z))$. Because $h^{0}(n x)=2$, one has that

$$
x \in W_{n}^{1} \backslash W_{n}^{2} .
$$

In particular, $x$ is a smooth point on $W_{n}^{1}$ and

$$
\operatorname{dim}_{x}\left(W_{n}^{1}\right)=2 g+n-3
$$

This is proved in [21] for the universal family of Teichmüller surfaces. But this family is étale over our family $\not h$ (see [13]), hence it is also valid in our situation. Let $U=\operatorname{Spec}(A)$ be a smooth affine neighbourhood of $x$ on $W_{n}^{1}$. We write $q$ for the restriction of $\not h$ to $\not^{-1}(U)$ and we write $Y$ instead of $h^{-1}(U)$. We also write $s$ for the restriction of $s$ to $U$. Hence we obtain a family of smooth curves of genus $g$

$$
q: Y \rightarrow U
$$

where $U$ is smooth of dimension $2 g+n-3$ and $q$ has a section $s$ such that, for each $z \in U$, one has

$$
h^{0}(n s(z))=2
$$

It is enough to prove that

$$
\operatorname{dim}(Z \cap U) \geq n+s+g-4-e .
$$

Let

$$
D:=s(U),
$$

a divisor on $Y$. Because of (2.3) we have that, for each $z \in U$

$$
h^{0}\left(q^{-1}(z),\left(\mathcal{O}_{Y}(n D)\right)(z)\right)=2
$$

[here $\left.\left(\mathcal{O}_{Y}(n D)\right)(z):=\mathcal{O}_{Y}(n D) \otimes_{\mathcal{O}_{Y}} \mathcal{O}_{q^{-1}(z)}\right]$. Because of Grauert's Theorem (see [15], Chapter III, Corollary $(12.9)), q_{*}\left(\mathcal{O}_{Y}(n D)\right)$ is a locally free $\mathcal{O}_{U}$-Module of rank 2 and, because of the Base Change Theorem (see [15], 
Chapter III, Theorem (12.11)), we have that

$$
\left(q_{*}\left(\mathcal{O}_{Y}(n D)\right)\right)(z) \cong H^{0}\left(q^{-1}(z),\left(\mathcal{O}_{Y}(n D)\right)(z)\right)
$$

for each $z \in U$ [i.e. there exists a natural morphism

$$
\left(q_{*}\left(\mathcal{O}_{Y}(n D)\right)\right)(z) \rightarrow H^{0}\left(q^{-1}(z),\left(\mathcal{O}_{Y}(n D)\right)(z)\right)
$$

and this is an isomorphism; here $\left(q_{*}\left(\mathcal{O}_{Y}(n D)\right)\right)(z)$ means $q_{*}\left(\mathcal{O}_{Y}(n D)\right)$ $\otimes_{\mathcal{O}_{U}} \mathbf{C}(z)$ ]. Let $\tau$ be a global section of $\mathcal{O}_{Y}(d D)$ corresponding to the divisor $d D$. Then $\tau$ is also a global section of $q_{*}\left(\mathcal{O}_{Y}(d D)\right)$.

Let

$$
M=\mathcal{O}_{Y}(s D) \text { and } F=q_{*}(M) .
$$

Let

$$
1=h^{0}(M(x))=e+1+\varepsilon \quad \text { and } \quad i=h^{1}(M(x))=g+e+\varepsilon-s .
$$

By shrinking $Y$ if necessary, we can find two free $A$-modules $K^{0}$ and $K^{1}$ of rank resp. 1 and $i$ and an $A$-module-homomorphism $\alpha: K^{0} \rightarrow K^{1}$ such that, for each morphism $\phi: V \rightarrow U$ between affine sets we have that

$$
H^{0}\left(Y \times_{U} V, M_{V}\right) \cong \operatorname{ker}\left(\alpha \otimes_{A} \Gamma\left(V, \mathcal{O}_{V}\right)\right) .
$$

This follows from [24, Chapter V]. Hence the $A$-module-homomorphism $\alpha$ gives rise to a morphism $\tilde{\alpha}: \tilde{K}^{0} \rightarrow \tilde{K}^{1}$ between free $\mathcal{O}_{U}$-Modules such that $U \cap Z$ is an irreducible component of $\left\{z \in U: \Lambda^{\varepsilon}(\tilde{\alpha}(z))=0\right\}$ [for $\tilde{K}^{0}$ and $\tilde{K}^{1}$ see $\left[15\right.$, p. 110], by $\tilde{\alpha}(z)$ we mean $\left.\tilde{\alpha} \otimes_{\mathcal{O}_{U}} \mathbf{C}(z)\right]$. Hence, a lower bound for the dimension of $Z$ can be obtained from this description. But, for each $z \in U \cap Z$, one already has that $\Lambda^{\varepsilon+1}(\tilde{\boldsymbol{\alpha}}(z))=0$. Hence, the bound will not be as sharp as possible.

Consider

$$
\gamma^{\prime}:\left(q_{*}\left(\mathcal{O}_{Y}(n D)\right)\right)^{\otimes e} \rightarrow F
$$

which maps a local section $x_{1} \otimes \cdots \otimes x_{e}$ to $x_{1} \cdots x_{e} \cdot \tau$. This gives rise to

$$
\gamma:\left(q_{*}\left(\mathcal{O}_{Y}(n D)\right)\right)^{\otimes e} \rightarrow \tilde{K}^{0} .
$$

Let $K^{0 \prime}=\operatorname{coker}(\gamma(U))$. Because $m(\gamma(U)) \subset \operatorname{ker}(\alpha)$, this induces

$$
\alpha^{\prime}: K^{0 \prime} \rightarrow K^{1} \text {. }
$$

One can prove that $\tilde{K}^{0 \prime}$ is locally free of rank $\varepsilon$ and that for each $z \in U$, one has that

$$
h^{0}(M(z))-(e+1)=\operatorname{dim}\left(\operatorname{ker}\left(\tilde{\boldsymbol{\alpha}}^{\prime}(z)\right)\right) .
$$


The proof of those claims is exactly the same as the proof of Proposition 5.7 (namely the continuation) in [9]. Hence $U \cap Z$ is an irreducible component of $\left\{z \in U: \Lambda^{\varepsilon}\left(\tilde{\alpha}^{\prime}(z)\right)=0\right\}$. Because $U$ is smooth and because $s \leq g+e$, we can apply Corollary 11(i) of [18] to obtain that

$$
\operatorname{codim}_{U}(Z) \leq e+\varepsilon+g-s-\varepsilon+1=e+g+1-s .
$$

Hence

$$
\operatorname{dim}(Z) \geq 2 g+n-3-e-g-1+s=g+n+s-4-e
$$

which gives us (2.4), which was to be proved.

We are now able to prove the main result of this paper.

THEOREM 11. Let $Z$ be an irreducible component of $\dot{W}_{n, s}$ (see the Introduction). Let $2+e+\varepsilon=\min \left\{h^{0}(s z): z \in Z\right\}$. Assume that there exists $x \in Z$ such that

$$
h^{0}(s x)=2+e+\varepsilon \text { and }|s x| \text { is simple. }
$$

Then $\varepsilon=0$ and

$$
\operatorname{dim}(Z)=g+n+s-4-e
$$

Proof. It is not difficult to prove that the existence of $x$ implies the existence of a non-empty Zariski-open subset $U$ of $Z$ such that, for $t \in U$, one has

$$
h^{0}(s t)=2+e+\varepsilon \text { and }|s t| \text { is simple }
$$

(the proof is similar to the proof of Lemma (3.3) in [7]).

Each $t \in U$ gives rise to models $\Gamma_{t}$ on a rational surface $M$ as described in Construction 3. In order to obtain those models, one has to use some particular linear system $g_{s}^{2}$ (see Construction 1). For each $t \in U$, one has an $(e+\varepsilon-1)$-dimensional family of such linear systems. Because of Lemma 10, we have that

$$
\operatorname{dim}(U)=g+n+s-e-4+\eta
$$

with $\eta \geq 0$. Hence we obtain a $(g+n+s-5+\varepsilon+\eta)$-dimensional family of such linear systems $g_{s}^{2}$. This gives rise to a family of curves on a rational surface as obtained in Construction 3, whose dimension depends on the case obtained by a general such linear system. The result is summarized in Table 1. 


\begin{tabular}{|l|l|}
\hline \multicolumn{2}{|c|}{ TABLE 1 } \\
\hline a general $g_{s}^{2}$ gives rise to & $\begin{array}{l}\text { a lower bound for the dimension } \\
\text { of the family of curves is }\end{array}$ \\
\hline case A & $g+n+s+\varepsilon+\eta$ \\
case B1A & $g+n+s+\varepsilon+\eta-2$ \\
case B1B & $g+n+s+\varepsilon+\eta-2$ \\
case B2A & $g+n+s+\varepsilon+\eta-1$ \\
case B2B1 & $g+n+s+\varepsilon+\eta-2$ \\
case B2B2 & $g+n+s+\varepsilon+\eta-2$ \\
case B2C1 & $g+n+s+\varepsilon+\eta-1$ \\
case B2C2 & $g+n+s+\varepsilon+\eta-1$ \\
\hline
\end{tabular}

In it, we have to take care of the following considerations. Linear systems $g_{s}^{2}$, as obtained in Construction 1, give rise to 5-dimensional families of plane curves $\Gamma$ obtained in Construction 1 (choice of the base of the linear subspace of $H^{0}\left(p^{-1}(t), \mathcal{O}_{p^{-1}(t)}(s t)\right)$ corresponding to $\left.g_{s}^{2}\right)$. We also have to pay attention to Remark 4 . In each case, one obtains a family of curves $\tilde{\Gamma}$ on $M$ of dimension $d \geq g+1$. Because $g \geq 2$, it follows that

$$
d=\left(\tilde{\Gamma} .-K_{M}\right)+g-1 .
$$

This is proved in [8, Corollary 4]. The proof can be sketched as follows. Let $\Gamma$ be a general element of the obtained family of curves of geometric genus $g$ on $M$. Let $\phi^{\prime}: C \rightarrow \Gamma$ be the normalization of $\Gamma$. This induces a morphism $\phi: C \rightarrow M$ such that $\phi(C) \in \mathbf{P}$. This morphism gives rise to an injective morphism of sheaves

$$
d \phi: T_{C} \rightarrow \phi^{*}\left(T_{M}\right)
$$

(between tangent sheaves). Let $N_{\phi}$ be the Cokernel sheaf of $d \phi$. It follows from deformation theory of holomorphic maps (see [17]) that

$$
d \leq \operatorname{dim}\left(H^{0}\left(C, N_{\phi}\right)\right) .
$$

Let $Z$ be the ramification divisor of $\phi$ and let $N_{\phi}^{\prime}$ be the Cokernel sheaf of the injective morphism

$$
(d \phi)^{\prime}: T_{C}(Z) \rightarrow \phi^{*}\left(T_{M}\right) .
$$

From [3], Corollario (6.11), it follows that

$$
d \leq \operatorname{dim}\left(H^{0}\left(C, N_{\phi}^{\prime}\right)\right) .
$$

Hence, $\operatorname{dim}\left(H^{0}\left(C, N_{\phi}^{\prime}\right)\right)>g$, but $N_{\phi}^{\prime}$ is invertible, therefore $h^{1}\left(N_{\phi}^{\prime}\right)=0$. Hence $h^{1}\left(N_{\phi}\right)=0$ which means that infinitesimal deformations of $\phi$ are unobstructed (see [17] again). Because of this fact $N_{\phi}=N_{\phi}^{\prime}$ and

$$
h^{0}\left(N_{\phi}\right)=d \text {. }
$$


Because $N_{\phi}$ is invertible we have that

$$
N_{\phi} \cong \phi^{*}\left(-\omega_{M}\right) \otimes \omega_{C} .
$$

Hence, because of the Theorem of Riemann-Roch, we have

$$
h^{0}\left(N_{\phi}\right)=\left(-K_{M} \cdot \tilde{\Gamma}\right)+g-1 .
$$

From (2.9) and (2.10), (2.8) follows.

In the proof of Theorem 5 we computed $K_{M_{\varepsilon}}$. It follows that

$$
\begin{aligned}
\left(-K_{M_{\varepsilon}} \cdot \Gamma_{\varepsilon}\right) & =3 s-\sum\left(c_{i}: 0<i<\varepsilon\right)-s_{m-1} \\
& =3 s-\sum\left(a_{i} s_{i}: 1 \leq i \leq m-1\right) \\
& =3 s-n_{1}-n_{2}+s_{m-1}+s_{m}=s+n+s_{m-1}+s_{m} .
\end{aligned}
$$

Let us look at each specific case of Construction 3.

Case A. $K_{M} \in\left|\gamma_{0}^{*}\left(K_{M_{\varepsilon}}\right)+\sum\left(\gamma_{j}^{*}\left(E_{\varepsilon+j-1}\right): 2 \leq j \leq s_{m-1}\right)+E_{\varepsilon+s_{m-1}}\right|$. Hence, $\left(-K_{M} \cdot \tilde{\Gamma}\right)=\left(-K_{M_{\varepsilon}} \cdot \Gamma_{\varepsilon}\right)-s_{m-1}=s+n+1$.

Case B1. $K_{M_{\varepsilon+1}} \in\left|\left(\pi_{\varepsilon+1}\right) *\left(K_{M_{\varepsilon}}\right)+E_{\varepsilon+1}\right|$. Hence, $\left(-K_{M_{\varepsilon+1}} . \tilde{\Gamma}\right)=\left(-K_{M_{\varepsilon}} \cdot \Gamma_{\varepsilon}\right)-s_{m-1}=s+n$.

Case B1A. $K_{M} \in\left|\left(\pi_{\varepsilon+2} \circ \pi_{\varepsilon+3}\right) *\left(K_{M_{\varepsilon+1}}\right)+\left(\pi_{\varepsilon+3}\right)^{*}\left(E_{\varepsilon+2}\right)+E_{\varepsilon+3}\right|$. Hence, $\left(-K_{M} \cdot \tilde{\Gamma}\right)=\left(-K_{M_{\varepsilon+1}} \cdot \Gamma_{\varepsilon+1}\right)-1-1=s+n-2$.

Case B1B. $K_{m} \in\left|\left(\pi_{\varepsilon+2}\right) *\left(K_{M_{\varepsilon+1}}\right)-E_{\varepsilon+2}\right|$. Hence, $\left(-K_{M} . \tilde{\Gamma}\right)=\left(-K_{M_{\varepsilon+1}} . \Gamma_{\varepsilon+1}\right)-\rho=s+n-\rho$ with $\rho>1$. Hence, $\left(-K_{M} \cdot \tilde{\Gamma}\right) \leq s+n-2$.

Case B2. $K_{M_{\varepsilon+\alpha}} \in\left|\tau_{0}^{*}\left(K_{M_{\varepsilon}}\right)+\Sigma\left(\left(\tau_{i}\right)^{*}\left(E_{\varepsilon+i}\right): 0<i<\alpha\right)+E_{\varepsilon+\alpha}\right|$. Hence, $\left(-K_{M_{\varepsilon+\alpha}} \cdot \Gamma_{\varepsilon+\alpha}\right)=\left(-K_{M_{\varepsilon}} \cdot \Gamma_{\varepsilon}\right)-\kappa \alpha=s+n+s_{m-1}-\kappa \alpha$.

Case B2A. $\left(-K_{M} \cdot \tilde{\Gamma}\right)=\left(-K_{M_{\varepsilon+\alpha}} \cdot \Gamma_{\varepsilon+\alpha}\right)=s+n$.

Case B2B. $\left(-K_{M_{\varepsilon+\alpha}} \cdot \Gamma_{\varepsilon+\alpha}\right)=s+n$.

Case B2B1. $K_{M} \in\left|\left(\pi_{\varepsilon+\alpha+1}\right)^{*}\left(K_{M_{\varepsilon+\alpha}}\right)+E_{\varepsilon+\alpha+1}\right|$. Hence, $\left(-K_{M} \cdot \tilde{\Gamma}\right)=\left(-K_{M_{\varepsilon+\alpha}} \cdot \Gamma_{\varepsilon+\alpha}\right)-\rho=s+n-\rho$ with $\rho \geq 2$. Hence, $\left(-K_{M} . \tilde{\Gamma}\right) \leq s+n-2$.

Case B2B2.

$$
\begin{aligned}
K_{M} \in & \mid\left(\pi_{\varepsilon+\alpha+1} \circ \pi_{\varepsilon+\alpha+2}\right) *\left(K_{M_{\varepsilon+\alpha}}\right) \\
& +\left(\pi_{\varepsilon+\alpha+2}\right) *\left(E_{\alpha+\varepsilon+1}\right)+E_{\alpha+\varepsilon+2} \mid .
\end{aligned}
$$

Hence, $\left(-K_{M} \cdot \tilde{\Gamma}\right)=\left(-K_{M_{\varepsilon+\alpha}} \cdot \Gamma_{\varepsilon+\alpha}\right)-2=s+n-2$.

Case B2C. $K_{M_{\varepsilon+\alpha+1}} \in\left|\left(\pi_{\varepsilon+\alpha+1}\right) *\left(K_{M_{\varepsilon+\alpha}}\right)+E_{\varepsilon+\alpha+1}\right|$. Hence, $\left(-K_{M_{\varepsilon+\alpha+1}} \cdot \Gamma_{\varepsilon+\alpha+1}\right)=\left(-K_{M_{\varepsilon+\alpha}} \cdot \Gamma_{\varepsilon+\alpha}\right)-\beta=s+n$. 
Case B2C1. $K_{M} \in\left|\left(\pi_{\varepsilon+\alpha+2}\right)^{*}\left(K_{M_{\varepsilon+\alpha+1}}\right)+E_{\varepsilon+\alpha+2}\right|$. Hence, $\left(-K_{M} \cdot \tilde{\Gamma}\right)=\left(-K_{M_{\varepsilon+\alpha+1}}\right)-(\kappa-\beta)=s+n-(\kappa-\beta)$ with $\kappa-\beta$ $\geq 1$. Hence, $\left(-K_{M}\right.$. $\left.\tilde{\Gamma}\right) \leq s+n-1$.

Case B2C2. $K_{M} \in\left(\pi_{\varepsilon+\alpha+2}\right)^{*}\left(K_{M_{\varepsilon+\alpha+1}}\right)+E_{\varepsilon+\alpha+2} \mid$. Hence, $\left(-K_{M} \cdot \tilde{\Gamma}\right)=\left(-K_{M_{\varepsilon+\alpha+1}} \cdot \Gamma_{\varepsilon+\alpha+1}\right)-\beta=s+n-\beta$ with $\beta \geq 1$. Hence, $\left(-K_{M} \cdot \tilde{\Gamma}\right) \leq s+n-1$.

\begin{tabular}{|l|l|}
\hline \multicolumn{2}{|c|}{ TABLE 2} \\
\hline a general $g_{s}^{2}$ gives rise to & upper bound for $d$ \\
\hline case A & $s+n+g$ \\
case B1A & $s+n+g-3$ \\
case B1B & $s+n+g-3$ \\
case B2A & $s+n+g-1$ \\
case B2B1 & $s+n+g-3$ \\
case B2B2 & $s+n+g-3$ \\
case B2C1 & $s+n+g-2$ \\
case B2C2 & $s+n+g-2$ \\
\hline
\end{tabular}

Hence, using (2.8), we obtain an upper bound for $d$ as indicated by Table 2. Comparing Tables 1 and 2, we obtain that only case A (if $(n, s)=1$ ) and case $\mathrm{B} 2 \mathrm{~A}$ (if $(n, s) \neq 1$ ) are possible for a general $g_{s}^{2}$. Moreover, in those cases, it is also necessary that $\varepsilon=\eta=0$. Using (2.8), this proves the theorem.

Remark 12. Cases A and B2A are also the only cases in which the genus bound of Theorem 5 is attained.

\section{COROllaRY 13. Statement 8 follows immediately from Theorem 11.}

To conclude this section, we give an example to show that the asssumption " $|s x|$ is simple" is necessary for Theorem 11 to be true.

EXAmple 14. Let $g=14$ and let $\mathscr{M}^{\prime} \subset \mathscr{M}$ be the subset of the points on $\mathscr{M}$ corresponding to curves $C$ which are coverings $f: C \rightarrow E$ of degree 3 with a total ramification point $p$ of some elliptic curve. It is clear that both 6 and 9 are non-gaps of $p$. Because 9 is a non-gap of $p$ one has that 2 is a gap of $p(g>7)$. If 4 would be a non-gap of $p$, then each integer $m \geq 12$ would be a non-gap of $p$, which is impossible and if 5 would be a non-gap of $p$, then each integer $m \geq 14$ would be a non-gap of $p$. We prove that 3 is a gap of $p$. Assume that 3 is a non-gap of $p$, hence $\operatorname{dim}(|3 p|)=1$. Let $s: C \rightarrow \mathbf{P}^{1}$ be some associated trigonal covering of $C$. Because of Clifford's Theorem (see e.g. [15], Chapter IV, Theorem (5.4)), 
one has that $\operatorname{dim}(|6 p|)=2$, hence each divisor $D$ of $|6 p|$ equals $D_{1}+D_{2}$ for some $D_{1}, D_{2} \in|3 p|$. Let $f(p)=p^{\prime}$ and let $\phi: E \rightarrow \mathbf{P}^{1}$ be a double covering such that $p^{\prime}$ is a ramification point of $\phi$. Then $g=\phi \circ f$ : $C \rightarrow \mathbf{P}^{1}$ is a covering of degree 6 such that $p$ is a total ramification point of $g$. Hence $g$ corresponds to some linear system $g_{6}^{1} \subset|6 p|$, hence there exists a morphism $\psi: \mathbf{P}^{1} \rightarrow \mathbf{P}^{1}$ of degree 2 such that $\phi \circ f=\psi \circ s$.

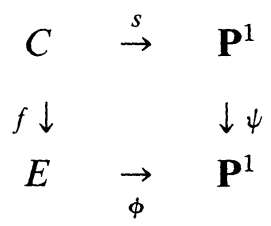

It is not difficult to see that, if $a$ is a general point on $\mathbf{P}^{1}$ then $f\left(s^{-1}(a)\right)$ contains 2 points. But then the diagram induces a morphism $\lambda^{\prime}: C \rightarrow$ $\mathbf{P}^{1} \times{ }_{\mathbf{P}^{1}} E$ (fibred product of $\psi$ and $\phi$ as defined in [15], p. 87) which is dominant, hence surjective. Hence $\mathbf{P}^{1} \times_{\mathbf{P}^{1}} E$ is irreducible. Let $C^{\prime}$ be the normalization of $\mathbf{P}^{1} \times \mathbf{p}^{1} E$. This gives rise to coverings $\lambda: C \rightarrow C^{\prime}$ and $\tau$ : $C^{\prime} \rightarrow \mathbf{P}^{1}$ such that $\tau$ has degree 4 and $\tau \circ \lambda=\psi \circ s$ has degree 6 , which is impossible.

This proves that $p \in \stackrel{\circ}{W}_{6,9} \subset \mathscr{H}$. On the other hand, $\operatorname{dim}\left(\mathscr{M}^{\prime}\right)=25$, hence $\stackrel{\circ}{W}_{6,9}$ contains some irreducible component $Z$ with $\operatorname{dim}(Z) \geq 25$, which is greater than 24 . Nevertheless, 14 satisfies the genus bound of Theorem 11.

[One can also prove that 9 is the second non-gap for a general point $p$ of $Z$. Indeed, if 7 would be a non-gap of $p$ then $p \in \mathscr{W}_{6,7}$-because $(6,7)=1$-but $\operatorname{dim}\left(\mathscr{W}_{6,7}\right)=22<25$. If 8 would be a non-gap of $p$ then - because $(8,9)=1-|9 p|$ would be simple and we obtain a contradiction to Theorem 11. Finally, it is easy to see that $p$ comes from a covering of an elliptic curve as considered above using the morphism $\chi: p^{-1}(\not p(p))$ $\rightarrow \mathbf{P}^{2}$ associated to $|9 p|$. Hence $\operatorname{dim}(Z)=25$.]

3. $\mathscr{W}_{n, s}$ is not empty (whenever possible). If $x \in \mathscr{W}_{n, s}$ and if $C=$ $p^{-1}(\not(x))$ and if $p$ is the point on $C$ corresponding to $x$. From Construction 1 , we obtain a plane model $\Gamma$ of $C$ with a singular point $q$ corresponding to $p$. In Construction 3, we obtain a birational morphism $\nu: M \rightarrow \mathbf{P}^{2}$ such that $\tilde{\Gamma}$, the strict transform of $\Gamma$, belongs to some definite linear system $\mathbf{P}$ on $M$. It is noted in Remark 12 that

$$
p_{a}(\tilde{\Gamma})=((n-1)(s-1)+1-(n, s)) / 2
$$

exactly in the Cases A and B2A. Let $\mathscr{E}=\nu^{-1}(q)$. We also have that $(\mathscr{E} . \tilde{\Gamma})=1$ in the cases A and B2A [we consider $\nu^{-1}(q)$ as a reduced divisor on $M]$. Hence in those cases $\mathscr{E} \cap \tilde{\Gamma}$ consists of exactly one point - say $\tilde{q}$ - which is a smooth point on $\tilde{\Gamma}$. 
From now on, starting with a point $q$ on $\mathbf{P}^{2}$ and a line $T$ on $\mathbf{P}^{2}$ through $q$, let $\tilde{M}$ be the surface mentioned above and let $\tilde{\mathbf{P}}$ be the linear system on $\tilde{M}$ mentioned above (which can be defined using the curves $L$ and $E_{l}$ mentioned in Construction 3), i.e. we consider Construction 3 without having the curve $\Gamma$, which is possible because the points at which we are blowing-up can be defined without $\Gamma$ in Cases A and B2A. We are going to prove that $\tilde{\mathbf{P}}$ indeed contains curves $\tilde{\Gamma}$ as mentioned above coming from a point $x$ on $\mathscr{W}_{n, s}$. As a first step, we prove the following theorem.

THEOREM 15. For each $0 \leq g \leq((s-1)(n-1)+1-(n, s)) / 2$, there exists an irreducible element $\Gamma$ of $\tilde{\mathbf{P}}$ such that $\Gamma$ has geometric genus $g$.

(The main line of the following proof is also suggested to me by H. C. Pinkham.)

LEMMA 16. It is enough to prove that there exists an irreducible element $\Gamma_{0}$ of $\tilde{\mathbf{P}}$ of geometric genus 0 which has ordinary nodes as its only singularities.

Proof. Because $p_{a}\left(\Gamma_{0}\right)=((s-1)(n-1)+1-(n, s)) / 2$, the curve $\Gamma_{0}$ has precisely $\delta=((s-1)(n-1)+1-(n, s)) / 2$ ordinary nodes - say $s_{1}, \ldots, s_{\delta}$. Let $\bar{\delta}=\delta-g$. Because $\left(K_{\tilde{M}} \cdot \Gamma_{0}\right)<0$, it follows from $[28((2.3) \mathrm{V})]$ that $\tilde{\mathbf{P}}$ contains smooth irreducible elements, hence we can apply [28, Theorem (2.13)]. It follows from that theorem that, for any choice of $\bar{\delta}$ nodes of $\Gamma_{0}$ - say $s_{1}, \ldots, s_{\bar{\delta}}$ - there exist a smooth curve $T$ and a family of effective divisors $\eta \subset \tilde{M} \times T$ over $T$ such that there exist $t_{0} \in T$ and sections $S_{1}, \ldots, S_{\bar{\delta}}$ of $\eta$ over $T$ such that

$$
\eta_{t_{0}}=\Gamma_{0} \quad \text { and } \quad S_{1}\left(t_{0}\right)=s_{1}, \ldots, S_{\bar{\delta}}\left(t_{0}\right)=s_{\bar{\delta}},
$$

if $t \in T \backslash\left\{t_{0}\right\}$ then $\eta_{t}$ is an irreducible element of $\tilde{\mathbf{P}} ; S_{1}(t), \ldots, S_{\bar{\delta}}(t)$ are ordinary nodes of $\eta_{t}$; if $x \in \eta_{t} \backslash\left\{S_{1}(t), \ldots, S_{\bar{\delta}}(t)\right\}$ then $x$ is a smooth point of $\eta_{T}$.

In particular, if $t \in T \backslash\left\{t_{0}\right\}$, then $\eta_{t}$ is an irreducible element of $\tilde{\mathbf{P}}$ of geometric genus $g$.

Now, we are going to prove that $\tilde{\mathbf{P}}$ contains rational curves. Then, we prove that the general one has ordinary nodes as its only singularities.

In Construction 1 we obtained particular linear systems $g_{s}^{2}$. Namely, there exists a point $p$ and a fixed-point-free linear system $g_{d}^{1}$ - say $\gamma$ - with $n p \in \gamma$ such that $(n-s) p+\gamma \subset g_{s}^{2}$ and $g_{s}^{2}$ is simple. We call them suited linear systems. 
We first investigate "how many" such suited linear systems $g_{s}^{2}$ exist on $\mathbf{P}^{1}$. Let $\mathscr{G}_{d}^{r}$ be the space parametrizing the linear systems $g_{d}^{r}$ on $\mathbf{P}^{1}$ and let $\mathscr{S} \subset \mathscr{G}_{s}^{2}$ be the locus of the suited linear systems.

LEMma 17. $\mathscr{S}$ is irreducible and has dimension $s+n-2$.

Proof. One has that $\mathscr{S} \subset \mathscr{S}^{\prime}$ with

$\mathscr{S}^{\prime}=\left\{g_{s}^{2} \in \mathscr{G}_{s}^{2}:\right.$ there exist $p \in \mathbf{P}^{1}$ and $\gamma \in \mathscr{G}_{n}^{1}$ such that $n p \in g$;

$g$ has no fixed points and $\left.g+(s-n) p \subset g_{s}^{2}\right\}$.

Below we prove that $\mathscr{S}^{\prime}$ is irreducible and has dimension $s+n-2$. Now

$$
\mathscr{S}=\mathscr{S}^{\prime} \cap\left\{g_{s}^{2} \in \mathscr{G}_{s}^{2}: g_{s}^{2} \text { is simple }\right\} .
$$

Because $\left\{g_{s}^{2} \in \mathscr{G}_{s}^{2}: g_{s}^{2}\right.$ is simple $\}$ is a non-empty Zariski-open subset of $\mathscr{G}_{s}^{2}$, we find that $\mathscr{S}$ is irreducible and has dimension $s+n-2$ if $\mathscr{S} \neq \varnothing$.

We construct an element of $\mathscr{S}$ as follows. Let $p \in \mathbf{P}^{1}$ and $D \in\left(\mathbf{P}^{1}\right)^{(d)}$ with $p \notin \operatorname{Supp}(D)$. Let $D^{\prime} \in\left(\mathbf{P}^{1}\right)^{(s)}$ with $\operatorname{deg}\left(\inf \left(D, D^{\prime}\right)\right)=1$ and $p \notin$ $\operatorname{Supp}\left(D^{\prime}\right)$. Let $\gamma=\langle D, n p\rangle \in \mathscr{G}_{n}^{1}$ and $g_{s}^{2}=\left\langle\gamma+(s-n) p, D^{\prime}\right\rangle$. Clearly, $g_{s}^{2} \in \mathscr{S}^{\prime}$ and $g_{s}^{2}$ is simple because $\operatorname{deg}\left(\inf \left(D+(s-n) p, D^{\prime}\right)\right)=1$, while both $D+(s-n) p$ and $D^{\prime}$ belong to $g_{s}^{2}$.

We now prove the irreducibility and compute the dimension of $\mathscr{S}^{\prime}$. Let $I \subset \mathscr{G}_{n}^{1} \times \mathbf{P}^{1}$ be defined by

$$
(\gamma, p) \in I \text { if and only if } n p \in \gamma .
$$

Consider the projection morphism $\pi_{1}: I \rightarrow \mathbf{P}^{1}$. Clearly, for $p \in \mathbf{P}^{1}$, $\left(\pi_{1}\right)^{-1}(p)$ is irreducible of dimension $n-1$, hence $I$ is irreducible of dimension $n$. Let $\left(\mathscr{G}_{n}^{1}\right)^{\prime}=\left\{\gamma \in \mathscr{G}_{n}^{1}: \gamma\right.$ has no fixed points $\}$. This is a Zariski-open subset of $\mathscr{G}_{n}^{1}$ and $I^{\prime}=I \cap\left(\left(\mathscr{G}_{n}^{1}\right)^{\prime} \times \mathbf{P}^{1}\right)$ is a non-empty Zariski-open subset of $I$. Let $J \subset \mathscr{G}_{s}^{2} \times I^{\prime}$ be defined by

$$
(h,(\gamma, p)) \in J \text { if and only if } \gamma+(s-n) p \subset h .
$$

Consider the projection morphism $\pi_{2}: J \rightarrow I^{\prime}$. Clearly, for $(\gamma, p) \in I^{\prime}$, $\left(\pi_{2}\right)^{-1}((\gamma, p))$ is irreducible of dimension $s-2$ [namely, if $(\gamma, p) \in I^{\prime}$, then

$$
\begin{aligned}
\left(\mathbf{P}^{1}\right)^{(s)} \backslash(\gamma+(s-n) p) \rightarrow\left(\pi_{2}\right)^{-1}((\gamma, p)): & \\
D & \rightarrow(\langle\gamma+(s-n) p, D\rangle,(\gamma, p))
\end{aligned}
$$

is surjective with 2-dimensional fibres - the fibre over $(h,(\gamma, p))$ being $h \backslash(\gamma+(n-s) p)$ ], hence $J$ is irreducible of dimension $s+n-2$. Consider the projection morphism $\pi_{3}: J \rightarrow \mathscr{G}_{s}^{2}$. Clearly $\pi_{3}(J)=\mathscr{S}^{\prime}$ and it 
is easy to see that $\pi_{3}$ is generically injective. Hence $\mathscr{S}^{\prime}$ is irreducible and has dimension $s+n-2$.

COROllaRy 18. If $(n, s)=1$, then $\tilde{\mathbf{P}}$ contains rational elements.

Proof. Indeed, there exist suited linear systems $g_{s}^{2}$ on $\mathbf{P}^{1}$ and Case A only occurs.

If $(n, s) \neq 1$, then we are going to prove that we obtain Case B2A in Construction 3 if $g$ is a general element of $\mathscr{S}$. This is done to prove the following Lemma.

Lemma 19. If $(n, s) \neq 1$, then $\tilde{\mathbf{P}}$ contains rational elements.

Proof. Let $g_{s}^{2}$ be a general element of $\mathscr{S}$. This gives rise to a 5-dimensional family of generically injective morphisms $\phi: \mathbf{P}^{1} \rightarrow M$ with $\phi\left(\mathbf{P}^{1}\right) \in \mathbf{P}$ as obtained in Construction 3 for some B-Case. Each $\psi \in$ $\operatorname{Aut}\left(\mathbf{P}^{1}\right)$ gives rise to such a morphism $\psi^{*}(\phi)=\phi \circ \psi$ which is associated to $\psi^{*}\left(g_{s}^{2}\right) \in \mathscr{S}$. Moreover, $\operatorname{dim}\left(\operatorname{Aut}\left(\mathbf{P}^{1}\right)\right)=3$. Combining those considerations with Lemma 17, gives rise to Table 3. This table gives the lower bound for the dimension of some irreducible subset of $\mathbf{P}$ consisting of irreducible rational elements, under the assumption that a general $g_{s}^{2}$ gives rise to the B-Case under consideration (and, of course do not forget Remark 4). Comparing this with Table 2, we see that a general $g_{s}^{2} \in \mathscr{S}$ gives rise to Case $\mathrm{B} 2 \mathrm{~A}$.

We already obtained that $\tilde{\mathbf{P}}$ contains an irreducible subset $\tilde{\mathbf{P}}(0)$ consisting of irreducible rational elements with

$$
\begin{array}{ll}
\operatorname{dim}(\tilde{\mathbf{P}}(0))=s+n & \text { if }(n, s)=1 \\
\operatorname{dim}(\tilde{\mathbf{P}}(0))=s+n-1 & \text { if }(n, s) \neq 1 .
\end{array}
$$

We are going to prove that, if $\Gamma_{0}$ is a general element of $\tilde{\mathbf{P}}(0)$, then $\Gamma_{0}$ has ordinary nodes as its only singularities. If this is proved, then Theorem 15 holds because of Lemma 16.

\begin{tabular}{|l|l|}
\hline \multicolumn{2}{|c|}{ TABLE 3 } \\
\hline Case & Lower bound \\
\hline B1A & $s+n-2$ \\
B1B & $s+n-2$ \\
B2A & $s+n-1$ \\
B2B1 & $s+n-2$ \\
B2B2 & $s+n-2$ \\
B2C1 & $s+n-1$ \\
B2C2 & $s+n-1$ \\
\hline
\end{tabular}


Proposition 20. If $\Gamma_{0}$ is a general element of $\tilde{\mathbf{P}}(0)$, then $\Gamma_{0}$ has ordinary nodes as its only singularities.

Proof. As noted at the beginning of this section,

$$
\operatorname{Sing}\left(\Gamma_{0}\right) \subset \tilde{M} \backslash \mathscr{E} .
$$

But $\left.\nu\right|_{\tilde{M} \backslash \mathscr{E}}: \tilde{M} \backslash \mathscr{E} \rightarrow \mathbf{P}^{2} \backslash\{q\}$ is an isomorphism (blowing-up). Let $\phi:$ $\mathbf{P}^{1} \rightarrow \tilde{M}$ be a generically injective morphism such that $\phi\left(\mathbf{P}^{1}\right)=\Gamma_{0}$ is a general element of $\tilde{\mathbf{P}}(0)$. It is shown in the proof of Theorem 11 that $\Gamma_{0}$ has no cusps. Hence $N_{\phi}$, the Cokernel of the injection $T_{\mathbf{P}^{1}} \rightarrow \phi^{*}\left(T_{\tilde{M}}\right)$ is an invertible sheaf on $\mathbf{P}^{1}$ with

$$
\begin{array}{ll}
\operatorname{deg}\left(N_{\phi}\right)=s+n+1 & \text { if }(n, s)=1 \\
\operatorname{deg}\left(N_{\phi}\right)=s+n & \text { if }(n, s) \neq 1 .
\end{array}
$$

Moreover, $H^{0}\left(\mathbf{P}^{1}, N_{\phi}\right)$ corresponds in a natural way to the space of the first order deformations of $\phi$ (modulo $\operatorname{Aut}\left(\mathbf{P}^{1}\right)$ ) (see [17]) and because $H^{1}\left(\mathbf{P}^{1}, N_{\phi}\right)=0$, there is no obstruction.

If $x$ is a singular point of $\Gamma_{0}$, then $x \in \tilde{M} \backslash \mathscr{E} \cong \mathbf{P}^{2} \backslash\{q\}$. Assume that the multiplicity of $x$ is equal to $m \geq 3$. Then $m \leq s-n$ and there exist $q_{1}, \ldots, q_{m} \in \mathbf{P}^{1}$ with

$$
\phi\left(q_{1}\right)=\cdots=\phi\left(q_{m}\right)=x .
$$

Because $H^{1}\left(\mathbf{P}^{1}, N_{\phi}\left(-q_{1}-\cdots-q_{m}\right)\right)=0$, there exists $\sigma \in H^{0}\left(\mathbf{P}^{1}, N_{\phi}\right)$ with $\sigma\left(q_{1}\right) \neq 0$ and $\sigma\left(q_{2}\right)=\cdots=\sigma\left(q_{m}\right)=0$. Then $\sigma$ gives rise to a global deformation of $\Gamma_{0}$ in $\tilde{\mathbf{P}}(0)$ which splits the singular point $x$ of $\Gamma_{0}$ into singular points of lower multiplicity - a contradiction to the assumption that $\Gamma_{0}$ is general. Let $q_{1}, q_{2} \in \mathbf{P}^{1}$ with $\phi\left(q_{1}\right)=\phi\left(q_{2}\right)=x$. Because $H^{1}\left(\mathbf{P}^{1}, N_{\phi}\left(-q_{1}-q_{2}\right)\right)=0$, there exists $\sigma \in H^{0}\left(\mathbf{P}^{1}, N_{\phi}\right)$ with $\sigma\left(q_{1}\right) \neq 0$ and $\sigma\left(q_{2}\right)=0$. If the two branches of $\Gamma_{0}$ at $x$ have a contact of order $b \geq 2$, then $\sigma$ gives rise to a global deformation of $\Gamma_{0}$ which splits $x$ into double points where the two branches have a contact of order lower than $b$ - again a contradiction to the assumption that $\Gamma_{0}$ is general. Hence, the only singularities of $\Gamma_{0}$ are ordinary nodes. (For more details concerning those arguments, see [2], p. 97 and 98.)

One can also prove Proposition 20 using [3, Corollario (6.11)] in a way similar to the proof of (2.b) and (2.c) in [6].

Corollary 21. For each $0 \leq g \leq((s-1)(n-1)+1-(n, s)) / 2$, there exists a curve $C$ of genus $g$ such that $C$ contains a point $p$ such that both $n$ and $s$ are non-gaps of $p$ and $|s p|$ is simple. 
Proof. Let $\Gamma$ be an irreducible element of $\tilde{\mathbf{P}}$ of geometric genus $g$. Consider the linear system $\mathscr{L}$ of the lines on $\mathbf{P}^{2}$ through $q$. Let $C$ be the normalization of $\Gamma$. Then $\nu^{*}(\mathscr{L})$ is a one-dimensional linear system on $\tilde{M}$. It is not difficult to see that $\nu^{*}(\mathscr{L})$. $\Gamma$ gives rise to a linear system $g_{s}^{1}$ on $C$-being a subsystem of $g_{s}^{2}$, the linear system corresponding to $\nu(\Gamma) \subset \mathbf{P}^{2}$ - such that there exists $p \in C$ with $s p \in g_{s}^{1}$ and $\{D-$ $\left.(s-n) p: \quad D \in g_{s}^{1}\right\}$ has no fixed points.

Now, we are going to prove that, for $((s-1)(n-1)+1-(n, s)) \geq$ $g \geq a^{\prime}(n-1)+b^{\prime}$ with $s=a^{\prime} n+b\left(0<b^{\prime}<n\right)$, there exists a couple $(C, p)$ as in Corollary 21 such that $n$ is the first non-gap of $p$ and $s$ is the first non-gap of $p$ which is not a multiple of $n$.

Let $\Gamma$ be an irreducible element of $\tilde{\mathbf{P}}$ of geometric genus $g$ and with ordinary nodes $p_{1}, \ldots, p_{\delta}$ as its only singularities. Let $\nu^{\prime}: \tilde{M}^{\prime} \rightarrow \tilde{M}$ be the blowing-up of $\tilde{M}$ at the points $p_{1}, \ldots, p_{\delta}$ and let $F_{1}, \ldots, F_{\delta}$ be the exceptional divisors on $\tilde{M}^{\prime}$ such that $\nu^{\prime}\left(F_{i}\right)=\left\{p_{i}\right\}$ for each $1 \leq i \leq \delta$. Let $\Gamma^{\prime}$ be the strict transform of $\Gamma$ on $\tilde{M}^{\prime}$, hence

$$
\Gamma^{\prime}=\left|\left(\nu^{\prime}\right) *(\Gamma)-\sum\left(2 F_{i}: 1 \leq i \leq \delta\right)\right| .
$$

Then $\Gamma^{\prime}$ is a smooth curve of genus $g$ on $\tilde{M}^{\prime}$. Consider the exact sequence

$$
0 \rightarrow \omega_{\tilde{M}^{\prime}} \rightarrow \omega_{\tilde{M}^{\prime}} \otimes_{\tilde{O}_{\tilde{M}^{\prime}}} \mathcal{O}_{\tilde{M}^{\prime}}\left(\Gamma^{\prime}\right) \rightarrow\left(\omega_{\tilde{M}^{\prime}} \otimes_{\mathcal{O}_{\tilde{M}^{\prime}}} \mathcal{O}_{\tilde{M}^{\prime}}\left(\Gamma^{\prime}\right)\right) \otimes_{\mathscr{O}_{\tilde{M}^{\prime}}} \mathcal{O}_{\Gamma^{\prime}} \rightarrow 0 .
$$

Because $\tilde{M}^{\prime}$ is a rational surface, we obtain that this induces an isomorphism

$$
H^{0}\left(\tilde{M}^{\prime}, \omega_{\tilde{M}^{\prime}} \otimes_{\mathcal{O}_{\tilde{M}^{\prime}}} \mathcal{O}_{\tilde{M}^{\prime}}\left(\Gamma^{\prime}\right)\right) \rightarrow H^{0}\left(\tilde{M},\left(\omega_{\tilde{M}^{\prime}} \otimes_{\tilde{O}_{\tilde{M}^{\prime}}} \mathcal{O}_{\tilde{M}^{\prime}}\left(\Gamma^{\prime}\right)\right) \otimes_{\mathcal{O}_{\bar{M}^{\prime}}} \mathcal{O}_{\Gamma^{\prime}}\right)
$$

and because of the Adjunction Formula, we obtain an isomorphism

$$
H^{0}\left(\tilde{M}^{\prime}, \omega_{\tilde{M}^{\prime}} \otimes_{\tilde{O}_{\tilde{M}^{\prime}}} \mathcal{O}_{\tilde{M}^{\prime}}\left(\Gamma^{\prime}\right)\right) \rightarrow H^{0}\left(\Gamma^{\prime}, \omega_{\Gamma^{\prime}}\right) \text {. }
$$

This gives rise to an isomorphism

$$
\left|K_{\tilde{M}^{\prime}}+\Gamma^{\prime}\right| \rightarrow\left|K_{\Gamma^{\prime}}\right|: D \rightarrow D . \Gamma^{\prime} .
$$

But

$$
\left|K_{\tilde{M}^{\prime}}\right|=\left|\left(\nu^{\prime}\right) *\left(K_{\tilde{M}}\right)+\sum\left(F_{i}: 1 \leq i \leq \delta\right)\right|
$$

hence

$$
\left|K_{\tilde{M}^{\prime}}+\Gamma^{\prime}\right|=\left|\left(\nu^{\prime}\right) *\left(K_{\tilde{M}^{\prime}}+\Gamma\right)-\sum\left(F_{i} 1 \leq i \leq \delta\right)\right| .
$$

Therefore

$$
\begin{aligned}
\nu\left(\left|K_{\tilde{M}^{\prime}}+\Gamma^{\prime}\right|\right) & \\
& =\left\{D \in\left|K_{\tilde{M}}+\Gamma\right|: p_{\imath} \in \operatorname{Supp}(D) \text { for each } 1 \leq i \leq \delta\right\} .
\end{aligned}
$$


It follows that the projective space associated to

$$
\operatorname{ker}\left(H^{0}\left(\tilde{M}, \omega_{\tilde{M}} \otimes_{\mathscr{O}_{\tilde{M}}} \mathcal{O}_{\tilde{M}}(\Gamma)\right)\right) \rightarrow \mathbf{C}^{\delta}: s \rightarrow\left(s\left(p_{1}\right), \ldots, s\left(p_{\delta}\right)\right)
$$

is isomorphic to $\left|K_{\tilde{\Gamma}}\right|$. Let $p^{\prime} \in \Gamma^{\prime}$ such that $\left(\nu \circ \nu^{\prime}\right)\left(p^{\prime}\right)=q$. Because $n$ and $s$ are both non-gaps of $p^{\prime}$, one has that

$$
h^{0}\left(s p^{\prime}\right) \geq a^{\prime}+2
$$

and $h^{0}\left(s p^{\prime}\right)=a^{\prime}+2$ if and only if $n$ is the first non-gap of $p^{\prime}$ and $s$ is the first non-gap of $p^{\prime}$ which is not a multiple of $n$. Hence, in order to obtain our aim, it is enough to prove that there exists such a curve $\Gamma^{\prime}$ with $h^{0}\left(s p^{\prime}\right)=a^{\prime}+2$. Because of the Theorem of Riemann-Roch, this is equivalent to

$$
h^{1}\left(s p^{\prime}\right)=a^{\prime}+1+g-s .
$$

On the other hand, because $h^{0}\left(s p^{\prime}\right) \geq a^{\prime}+2$, we already know that $h^{1}\left(s p^{\prime}\right) \geq a^{\prime}+1+g-s$. Hence, because $g \geq a^{\prime}(n-1)+b^{\prime}=s-a^{\prime}$, we have that $h^{1}\left(s p^{\prime}\right) \geq 1$. Hence, using Serre-Duality, we obtain that $h^{0}\left(K_{\Gamma^{\prime}}-s p^{\prime}\right) \geq 1$. This proves that there exists some effective canonical divisor on $\Gamma^{\prime}$ containing $s p^{\prime}$ and in order to prove our aim, it is enough to prove that there exists such a curve $\Gamma^{\prime}$ such that the family of effective canonical divisors on $\Gamma^{\prime}$ containing $s p^{\prime}$ has dimension exactly $a^{\prime}+g-s$. Now, let $R$ be a line on $\mathbf{P}^{2}$ such that $R \cap \nu(\Gamma)$ contains exactly $s$ points - say $q_{1}, \ldots, q_{s}$. Because $\left(\nu^{\prime} \circ \nu\right)^{-1}\left(q_{1}\right)+\cdots+\left(\nu^{\prime} \circ \nu\right)^{-1}\left(q_{s}\right)$ is linearly equivalent to $s p^{\prime}$ on $\Gamma^{\prime}$ we have to prove that for some $\Gamma$ the dimension of the elements of $\left|K_{\tilde{M}}+\Gamma\right|$ containing $p_{1}, \ldots, p_{\delta}, \nu^{-1}\left(q_{1}\right), \ldots, \nu^{-1}\left(q_{s}\right)$ has dimension exactly $a^{\prime}+g-s$. Let $D$ be such an element of $\left|K_{\tilde{M}}+\Gamma\right|$. It follows from Construction 3 that, as a reduced curve on $\mathbf{P}^{2}, \nu(D)$ has degree at most $s-3$. But this curve has $s$ points in common with the line $R$ hence $R \subset \nu(D)$. Hence

$$
D-\nu^{*}(R) \in\left|K_{\tilde{M}}+\Gamma-\nu^{*}(R)\right|
$$

or $D-\nu^{*}(R)$ is an element of

$$
\begin{aligned}
\mid\left(\tau_{0}\right) *\left(\left(\mu_{\varepsilon}\right) *(s-4) L-\sum\left(\left(\mu_{\varepsilon-1}\right) *\left(c_{i}-1\right) E_{i}: 1\right.\right. & \leq i \leq \varepsilon-1) \\
& \left.-\left(s_{m-1}-1\right) E_{\varepsilon}\right) \mid,
\end{aligned}
$$

containing $p_{1}, \ldots, p_{\delta}$. Thus, we have to prove that the linear subsystem of $\left|K_{\tilde{M}}+\Gamma-\nu^{*}(R)\right|$ of elements containing $p_{1}, \ldots, p_{\delta}$ has dimension exactly $a^{\prime}+g-s$ for some curve $\Gamma$ as under considering.

We will use (and we are going to prove below) the following formula:

$$
\begin{aligned}
& \operatorname{dim}\left(\left|K_{\tilde{M}}+\Gamma-\nu^{*}(R)\right|\right) \\
& \quad=(((n-1)(s-1)+1-(n, s)) / 2)+a^{\prime}-s
\end{aligned}
$$


(notice that this formula gives our aim for

$$
g=((n-1)(s-1)+1-(n, s)) / 2) \text {. }
$$

THEOREM 22. Let

$$
a^{\prime}(n-1)+b^{\prime} \leq g \leq((n-1)(s-1)+1-(n, s)) / 2 .
$$

There exists a smooth curve $C$ of genus $g$ possessing a point $p$ such that $n$ is the first non-gap of $p$; $s$ is the first non-gap of $p$ which is not a multiple of $n$ and $|s p|$ is simple.

Proof. Because of the preceding arguments it is enough to prove that there exists an irreducible element $\Gamma$ of $\tilde{\mathbf{P}}$ such that $\Gamma$ has

$$
\delta=(((n-1)(s-1)+1-(n, s)) / 2)-g
$$

ordinary nodes - say $p_{1}, \ldots, p_{\delta}$ - and no other singularities, such that $S\left(p_{1}, \ldots, p_{\delta}\right)$, the linear subspace of $\left|K_{\tilde{M}}+\Gamma-\nu^{*}(L)\right|$ of elements containing $p_{1}, \ldots, p_{\delta}$, has dimension $a^{\prime}+g-s$.

Because of Lemma 19 and Proposition 20, $\tilde{\mathbf{P}}$ has an irreducible element $\Gamma_{0}$ of geometric genus 0 having $((n-1)(s-1)+1-(n, s)) / 2$ ordinary nodes (and no other singularities). We prove that we can find $p_{1}(0), \ldots, p_{\delta}(0)-\delta$ different nodes of $\Gamma_{0}$ - such that $S\left(p_{1}(0), \ldots, p_{\delta}(0)\right)$ has codimension $\delta$ in $\left|K_{\tilde{M}}+\Gamma-\nu^{*}(L)\right|$. This is clearly true for $\delta=0$. Assume that it is already proved for some $0 \leq \delta^{\prime}<\delta$ - hence there exist $p_{1}(0), \ldots, p_{\delta^{\prime}}(0)$ such that $S\left(p_{1}(0), \ldots, p_{\delta^{\prime}}(0)\right)$ has codimension $\delta^{\prime}$ in $\mid K_{\tilde{M}}$ $+\Gamma-\nu^{*}(L) \mid$. For each node $p$ of $\Gamma_{0}$ different from $p_{1}(0), \ldots, p_{\delta^{\prime}}(0)$ we have that

$$
\operatorname{codim}\left(S\left(p_{1}(0), \ldots, p_{\delta^{\prime}}(0), p\right)\right) \leq \delta^{\prime}+1
$$

and

$$
\operatorname{codim}\left(S\left(p_{1}(0), \ldots, p_{\delta^{\prime}}(0), p\right)\right)<\delta^{\prime}+1
$$

if and only if

$$
S\left(p_{1}(0), \ldots, p_{\delta^{\prime}}(0)\right)=S\left(p_{1}(0), \ldots, p_{\delta^{\prime}}(0), p\right)
$$

i.e. each element of $\left|K_{\tilde{M}}+\Gamma-\nu^{*}(L)\right|$ containing $p_{1}(0), \ldots, p_{\delta^{\prime}}(0)$ also contains $p$ (it concerns linear spaces!). Assume that for each such $p$ we have that

$$
\operatorname{codim}\left(S\left(p_{1}(0), \ldots, p_{\delta^{\prime}}(0), p\right)\right)=\delta^{\prime} .
$$

Then, each element of $\left|K_{\tilde{M}}+\Gamma-\nu^{*}(L)\right|$ containing $p_{1}(0), \ldots, p_{\delta^{\prime}}(0)$ also contains the other nodes of $\Gamma$, hence

$$
S\left(p_{1}(0), \ldots, p_{\delta^{\prime}}(0)\right)=S\left(\left\{p: p \text { is a node of } \Gamma_{0}\right\}\right) .
$$


But - from the reasonings done above - it follows that

$$
S\left(\left\{p: p \text { is a node of } \Gamma_{0}\right\}\right) \cong\left|K_{\mathbf{P}^{1}}-s x\right|
$$

with $x \in \mathbf{P}^{1}$. But $\left|K_{\mathbf{p}^{1}}-s x\right|=\varnothing$ hence it would follow that $S(\{p: p$ is a node of $\left.\left.\Gamma_{0}\right\}\right)=\varnothing$. Because "containing a point" is a linear condition for $H^{0}\left(\tilde{M}, \mathcal{O}_{\tilde{M}}\left(K_{\tilde{M}}+\Gamma-\nu^{*}(L)\right)\right)$ one has that this would imply that

$$
\operatorname{codim}\left(S\left(\left\{p: p \text { is a node of } \Gamma_{0}\right\}\right)\right)=\delta^{\prime}>\operatorname{dim}\left(\left|K_{\tilde{M}}+\Gamma-\nu^{*}(L)\right|\right) \text {. }
$$

From (3.10), it would follow that

$$
\delta^{\prime}>(((n-1)(s-1)+1-(n, s)) / 2)+a^{\prime}-s,
$$

hence

$$
-g>a^{\prime}-s \text { or } g<s-a^{\prime}=a^{\prime}(n-1)+b^{\prime},
$$

which is a contradiction to the assumption that $g \geq a^{\prime}(n-1)+b^{\prime}$. Hence there exists a node $p_{\delta^{\prime}+1}(0)$ of $\Gamma_{0}$ such that

$$
\operatorname{codim}\left(S\left(p_{1}(0), \ldots, p_{\delta^{\prime}+1}(0)\right)\right)=\delta^{\prime}+1
$$

and in particular there exist nodes $p_{1}(0), \ldots, p_{\delta}(0)$ of $\Gamma_{0}$ such that

$$
\operatorname{codim}\left(S\left(p_{1}(0), \ldots, p_{\delta}(0)\right)\right)=\delta .
$$

From [28] it follows that we can find a smooth curve $T$ and a family $\eta \subset \tilde{M} \times T$ of effective divisors on $\tilde{M}$ over $T$ with sections $S_{1}, \ldots, S_{\delta}$ of $\eta$ over $T$ such that there exists $t_{0} \in T$ such that $\eta_{t_{0}}=\Gamma_{0}$ and $S_{t}\left(t_{0}\right)=p_{i}(0)$; if $t \in T \backslash\left\{t_{0}\right\}$, then $\eta_{t}$ is an irreducible element of $\tilde{\mathbf{P}} ; S_{1}(t), \ldots, S_{\delta}(t)$ are ordinary nodes of $\eta_{t} ; \eta_{t}$ has no other singularities (see also the proof of Lemma 16). In particular, for $t \in T \backslash\left\{t_{0}\right\}, \eta_{t}$ has geometric genus $g$. Consider

$$
S\left(S_{1}, \ldots, S_{\delta}\right) \subset\left|K_{\tilde{M}}+\Gamma-\nu^{*}(L)\right| \times T
$$

with

$(D, t) \in S\left(S_{1}, \ldots, S_{\delta}\right)$ if and only if $D \in S\left(S_{1}(t), \ldots, S_{\delta}(t)\right)$.

This is a Zariski-closed subset of $\left|K_{\tilde{M}}+\Gamma-\nu^{*}(L)\right| \times T$. Each fibre of the projection morphism

$$
\pi: S\left(S_{1}, \ldots, S_{\delta}\right) \rightarrow T
$$

is irreducible (as a linear space).

Because $\operatorname{dim}\left(\pi^{-1}\left(t_{0}\right)\right)=g+a^{\prime}-s$, there exists a non-empty Zariski-open subset $U$ of $T$ such that, for each $t \in U$, one has that

$$
\operatorname{dim}\left(\pi^{-1}(t)\right) \leq g+a^{\prime}-s .
$$

Because one also has that $\operatorname{codim}\left(S\left(S_{1}(t), \ldots, S_{\delta}(t)\right)\right) \leq \delta$, it follows that, for $t \in U$ we have that

$$
\operatorname{dim}\left(\pi^{-1}(t)\right)=g+a^{\prime}-s .
$$


Hence, if $t \in U \backslash\left\{t_{0}\right\}$ and $C$ is the normalization of $\eta_{t}$, then $C$ is a curve with the desired properties.

REMARK 23. A well-known formula for an effective divisor $D$ on a rational surface $M$ is the following one:

$$
\operatorname{dim}(|D|)=p_{a}(D)-\left(K_{M} . D\right)-1 \text {. }
$$

This formula holds if $\operatorname{Supp}(D)$ is connected and $D=Y_{1}+\cdots+Y_{r}$ with $Y_{1}$ irreducible; $Y_{i} \neq Y_{j}$ for $i \neq j$ and $\left(Y_{i} \cdot K_{M}\right)<0$. (For $r=1$ this is proved in Lemma (2.2) of [28], the reduction to this case is easy - see [5, Lemma (0.1)].) From this formula, (3.10) follows immediately. Piteously I am not able to prove that $\left|K_{\tilde{M}}+\Gamma-\nu^{*}(L)\right|$ contains a suited element. Therefore, I have to do some nasty work in order to prove (3.10).

Proposition 24. Formula (3.10) holds.

Proof. At first, consider the case $\bar{s}=a^{\prime} n+1$ and $\bar{g}=\left((n-1) a^{\prime} n\right) / 2$. Let $C$ be a smooth element of $\overline{\tilde{\mathbf{P}}}$ (we use ${ }^{-}$to denote the case $(n, \bar{s})$ instead of $(n, s)$ in Construction 3). Let $\{p\}=\overline{\mathscr{E}} \cap C$. Then both $n$ and $\bar{s}$ are non-gaps of $p$. Because each integer $\alpha n+\beta\left(a^{\prime} n+1\right)$ with $\alpha, \beta \in \mathbf{Z}_{\geq 0}$ are non-gaps of $p$, and because $p$ has exactly $\bar{g}$ gaps, this gives each non-gap of $p$. It follows that

$$
\begin{aligned}
& h^{0}((n-1) p)=1 ; \quad h^{0}(n p)=2 ; \quad h^{0}\left(a^{\prime} n p\right)=a^{\prime}+1 ; \\
& h^{0}(\bar{s} p)=a^{\prime}+2 ; \quad h^{0}\left(\left(\left(a^{\prime}+1\right) n-1\right) p\right)=a^{\prime}+2 ; \\
& h^{0}\left(\left(a^{\prime}+1\right) n p\right)=a^{\prime}+3 .
\end{aligned}
$$

Let $\not h: \bar{X} \rightarrow \overline{\mathscr{M}}$ be for the case $\bar{g}$ as $\not$ is defined in the introduction for the case $g$. Let $\bar{x} \in \overline{\mathscr{X}}$ such that $C \cong z^{-1}(\not h(\bar{x}))$ and $p$ corresponds to $\bar{x}$ under this isomorphism. For $1 \leq b \leq n-1$ let $Z_{b}$ be an irreducible component containing $\bar{x}$ of

$$
\left\{x \in \bar{X}: h^{0}(n x)>2 \text { and } h^{0}\left(\left(a^{\prime} n+b\right) x\right)>a^{\prime}+2\right\} .
$$

Let $\bar{x}_{b}$ be a general point of $Z_{b}$. Using the Semi-Continuity Theorem (see [15], Chapter III, Theorem (12.8)) one can prove that (using (3.11))

$$
\begin{array}{ll}
h^{0}\left((n-1) \bar{x}_{b}\right)=1 ; & h^{0}\left(n \bar{x}_{b}\right)=2 ; \\
h^{0}\left(a^{\prime} n \bar{x}_{b}\right)=a^{\prime}+1 ; & h^{0}\left(\left(a^{\prime} n+b\right) \bar{x}_{b}\right)=a^{\prime}+2 .
\end{array}
$$

LEMMA 25. $\left|\left(a^{\prime} n+b\right) \bar{x}_{b}\right|$ is simple.

From this lemma, it follows that

COROllaRY 26. $a^{\prime} n+b$ is the first non-gap of $\bar{x}_{b}$ which is not $a$ multiple of $n$. 
Proof (of Corollary 26). Because of (3.12), it follows from Lemma 10 that

$$
\operatorname{dim}\left(Z_{b}\right) \geq \bar{g}+n+a^{\prime} n+b-4-a^{\prime} .
$$

Assume that $a^{\prime} n+\bar{b}$ is the first non-gap of $\bar{x}_{b}$ which is not a multiple of $n$ (for some $\bar{b}<b$ ) [this non-gap is at least $a^{\prime} n+1$ because $h^{0}\left(a^{\prime} n \bar{x}_{b}\right)=$ $\left.a^{\prime}+1\right]$. Because of Lemma 25, we can use Theorem 11 which gives us that

$$
\operatorname{dim}\left(Z_{b}\right) \leq \bar{g}+n+a^{\prime} n+\bar{b}-4-a^{\prime} .
$$

Combining (3.13) and (3.14), we obtain that $b=\bar{b}$, which proves the corollary.

Proof (of Lemma 25). Assume that the lemma is not true. Then the following situation can be obtained because $Z_{b} \neq Z_{1}$ for $b \neq 1$ (use Lemma 10 for $Z_{b}$ and Theorem 11 for $Z_{1}$ ). Let $R$ be a discrete valuation ring, let $m$ be the closed point of $\operatorname{Spec}(R)$ and let $o$ be the generic point of $\operatorname{Spec}(R)$. Let $\mathscr{P}: \mathscr{C} \rightarrow \operatorname{Spec}(R)$ be a smooth family of curves of genus $\bar{g}$ and let $\mathscr{S}$ be a section of $\mathscr{P}$ such that

$$
\mathscr{C}_{m}=C \text { and } \mathscr{S}(m)=p .
$$

Let $\tilde{C}$ be a smooth curve defined over $k(o)$ [the quotient field of $R$ ] and let $\tilde{q}$ be a point on $\tilde{C}$ defined over $k(o)$. Let $\tilde{F}: \mathscr{C}_{0} \rightarrow \tilde{C}$ be a morphism of degree $t$ defined over $k(o)$ with $\tilde{F}^{-1}(\tilde{q})=\mathscr{S}(o) ; t$ divides $a^{\prime} n+\bar{b}$ (for some $\bar{b} \leq b)$ and $h^{0}\left(\left(\left(a^{\prime} n+\bar{b}\right) / t\right) \tilde{q}\right)=a^{\prime}+2\left[\right.$ here $h^{0}\left(\left(\left(a^{\prime} n+\bar{b}\right) / t\right) \tilde{q}\right)$ comes from $\tilde{q} \times_{k(o)} \overline{k(o)} \in \tilde{C} \times_{k(o)} \overline{k(o)}$ where $\overline{k(o)}$ is the algebraic closure of $k(o)$ ]. Because of [20, $\$ 2$ Lemma 1], we can assume that there exists a smooth curve $\mathscr{P}^{\prime}: \mathscr{C}^{\prime} \rightarrow \operatorname{Spec}(R)$ and a $\operatorname{Spec}(R)$-morphism $\mathscr{F}^{\prime}$ : $\mathscr{C} \rightarrow \mathscr{C}^{\prime}$ such that

$$
\mathscr{C}_{o}^{\prime}=\tilde{C} \quad \text { and } \quad \mathscr{F}_{o}^{\prime}=\tilde{F} .
$$

Let $\mathscr{S}^{\prime}: \operatorname{Spec}(R) \rightarrow \mathscr{C}^{\prime}$ be the unique section of $\mathscr{P}^{\prime}$ such that $\mathscr{S}^{\prime}(o)=\tilde{q}$ (see the Valuative Criterion of Properness in [15, Chapter II, Theorem 4.7]). Because of the Semicontinuity Theorem (see [15, Chapter III, Theorem 12.8]), we have that

$$
h^{0}\left(\left(\left(a^{\prime} n+\bar{b}\right) / t\right) \mathscr{S}^{\prime}(m)\right) \geq a^{\prime}+2 \text {. }
$$

Moreover, we have that $\left(\mathscr{F}_{m}^{\prime}\right)^{-1}\left(\mathscr{S}^{\prime}(m)\right)=\{\mathscr{S}(m)\}=\{p\}$ because $\left(\mathscr{F}_{o}^{\prime}\right)^{-1}\left(\mathscr{S}^{\prime}(o)\right)=\{\mathscr{S}(o)\}$. But $\left|\left(a^{\prime} n+b\right) p\right|$ is simple and $\left|\left(a^{\prime} n+1\right) p\right|+$ $(\bar{b}-1) p=\left|\left(a^{\prime} n+\bar{b}\right) p\right| \supset\left(\mathscr{F}_{m}^{\prime}\right)^{-1}\left(\left|\left(\left(a^{\prime} n+\bar{b}\right) / t\right) \mathscr{S}^{\prime}(m)\right|\right) \quad$ [the equality comes from (3.11)] and both sides of the inclusion have dimension $a^{\prime}+2$ ((3.11) again), hence, because of (3.15), we obtain that

$$
\left|\left(a^{\prime} n+1\right) p\right|+(\bar{b}-1) p=\left(\mathscr{F}_{m}^{\prime}\right)^{-1}\left(\left|\left(\left(a^{\prime} n+\bar{b}\right) / t\right) \mathscr{S}^{\prime}(a)\right|\right),
$$

which is a contradiction to the fact that $\left|\left(a^{\prime} n+1\right) p\right|$ is simple. 
Continuation of the proof of Theorem 22. Using the irreducible component $Z_{b^{\prime}}$ and repeating the proof of Proposition 20 for the case of genus $\bar{g}$ instead of 0 , we obtain a locally closed irreducible subset $\tilde{\mathbf{P}}(\bar{g})$ of $\tilde{\mathbf{P}}$ such that each $\Gamma \in \tilde{\mathbf{P}}(\bar{g})$ is an irreducible curve of geometric genus $\bar{g}$ having $\bar{\delta}=((s-1)(n-1)+1-(n, s)) / 2-\bar{g}$ ordinary nodes as its only singularities. Let $\Gamma$ be such a general curve and let $p_{1}, \ldots, p_{\bar{\delta}}$ be its nodes. Let $C_{b^{\prime}}$ be the normalization of $\Gamma$ and let $\bar{x}_{b^{\prime}}$ be the special Weierstrass point on $C_{b^{\prime}}$ corresponding to $\mathscr{E} \cap \Gamma$. Because $h^{0}\left(\left(a^{\prime} n+b\right) \bar{x}_{b^{\prime}}\right)=a^{\prime}+2$ (3.12), the linear subsystem of $\left|K_{\tilde{M}}+\Gamma-\nu^{*}(L)\right|$ containing $p_{1}, \ldots, p_{\bar{\delta}}$ has dimension $\bar{g}+a^{\prime}-a^{\prime} n-b^{\prime}$. Hence

$$
\begin{aligned}
& \operatorname{dim}\left(\left|K_{\tilde{M}}+\Gamma-\nu^{*}(L)\right|\right) \leq \bar{g}+a^{\prime}-a^{\prime} n-b^{\prime}+\bar{\delta} \\
& =(((s-1)(n-1)+1-(n, s)) / 2)+a^{\prime}-s .
\end{aligned}
$$

On the other hand, if $\tilde{\Gamma}$ is a smooth element of $|\Gamma|$ and $\tilde{p}=\mathscr{E} \cap \tilde{\Gamma}$, then $h^{0}(s \tilde{p}) \geq a^{\prime}+2$. Hence

$$
\begin{aligned}
\operatorname{dim}\left(\left|K_{\tilde{M}}+\Gamma-\nu^{*}(L)\right|\right) \\
\geq(((s-1)(n-1)+1-(n, s)) / 2)+a^{\prime}-s .
\end{aligned}
$$

From (3.16) and (3.17), Formula (3.10) follows.

4. Existence of Weierstrass gap sequences. As already mentioned in the Introduction, it is an open problem in the theory of Weierstrass points to decide which gap sequences are Weierstrass gap sequences. In [4] it is shown that there are gap sequences which are not Weierstrass gap sequences. In some cases the existence of Weierstrass gap sequences is known.

- The only gap sequence of genus $g$ such that 2 is a non-gap is equal to $(1,3,5,7, \ldots, 2 g-1)$. A point $p$ on a smooth curve $C$ of genus $g$ has gap sequence $(1,3,5,7, \ldots, 2 g-1)$ if and only if $p$ is a ramification point of some double covering $C \rightarrow \mathbf{P}^{1}$. In particular $C$ is hyperelliptic.

- Each gap sequence such that 3 is a non-gap is a Weierstrass gap sequence (see [23]).

- Each gap sequence such that 4 is a non-gap is a Weierstrass gap sequence (see [19]).

- Each gap sequence $(1,2,3, \ldots, g-1, g+1)$ for $1 \leq l \leq g-1$ is a Weierstrass gap sequence (see [25], [27]).

- Each hyperordinary gap sequence (see the Introduction) is a Weierstrass gap sequence (see [8], [11], [27]).

- Each gap sequence of weight $w:=\sum\left(n_{i}-i: 1 \leq i \leq g\right) \leq g / 2$ is a Weierstrass gap sequence (see [12]). 
Also, some other results - stated in a ringtheoretical way - are obtained (see e.g. [29]). Here, we hope to deduce some new existence results.

Theorem 27. Let $n \in \mathbf{Z}_{\geq 2}$ and let $a \in \mathbf{Z}_{\geq 2}$ such that

$$
a(n-1) \leq g<(a+1)(n-1) .
$$

Let $m, q \in \mathbf{Z}_{\geq 0}$ such that

(1) $q=e m+b$ with $e \in \mathbf{Z}_{\geq 1}$ and $0 \leq b<m$,

(2) $n<m<2 n$,

(3) $\left\{\alpha m+\beta q<g+1+a: \alpha, \beta \in \mathbf{Z}_{\geq 0}\right\}$ contains at least a elements,

(4) $q \geq(e+1) n$,

(5) $g<m+q-1-e-[q /(e+1)]$ [entire part],

then there exists a couple $(C, p)$ such that $C$ is a smooth curve of genus $g$ and $p \in C$ and the non-gaps of $p$ are

(1) each $x \in \mathbf{Z}_{\geq g+a+1}$,

(2) each $\alpha m+\beta q$ with $\alpha, \beta \in \mathbf{Z}_{\geq 0}$.

Proof. Consider the universal family $\not x: \mathscr{X} \rightarrow \mathscr{M}$ and let $W_{n}$ be some irreducible component of $W_{n}^{1}$. As already mentioned in the Introduction, the gap sequence of a general point $x$ on $W_{n}$ is the hyperordinary gap sequence of genus with first non-gap equal to $n$. It follows that

$$
x \in\left\{z \in \mathscr{X}: h^{0}(m z)=2 \text { and } h^{0}(q z) \geq e+2\right\}
$$

(because of (2), (1) and (4)). Hence, there exists some irreducible component $W_{m, q}^{\prime}$ of $\stackrel{\circ}{W}_{m, q}$ with $\bar{W}_{n} \subset \bar{W}_{m, q}^{\prime}$. Because of Lemma 10 ,

$$
\operatorname{dim}\left(W_{m, q}^{\prime}\right) \geq m+q+g-4-e .
$$

Therefore, because of (5), a general point $\bar{x}$ of $W_{m, q}^{\prime}$ does not belong to $W_{n}$.

Let $\tilde{m}$ be the first non-gap of $\bar{x}$. In particular $\bar{W}_{m, q}^{\prime} \subset \bar{W}_{\tilde{m}}$. Because of (5), this is impossible if $\tilde{m} \leq[q /(e+1)]$, hence $\tilde{m}>q /(e+1)$. Because $h^{0}(q \bar{x}) \geq e+2$, there exists some non-gap of $\bar{x}$, at most equal to $q$, which is not a multiple of $\tilde{m}$. Let $\tilde{q}$ be the smallest non-gap of $\bar{x}$ which is not a multiple of $\tilde{m}$. Let $\tilde{q}=f \tilde{m}+h$ with $0<h<\tilde{m}$. If $|\tilde{q} \bar{x}|$ would not be simple, then $|\tilde{m} \bar{x}|$ is composite with some involution $\gamma_{\bar{x}}$. But, as it is done in the proof of Lemma 25, it would follow that $|n x|$ is composite with some involution $\gamma_{x}$. An easy parameter count shows that this is impossible (see [8], Chapter 2). It follows from Theorem 11 that

$$
\operatorname{dim}\left(W_{m, q}\right)=g+\tilde{m}+\tilde{q}-4-f .
$$


Using (4.1), this gives us that

$$
m+q-e \leq \tilde{m}+\tilde{q}-f .
$$

If $\tilde{q}=q$ and $\tilde{m}<m$, then $f<e$, hence $\tilde{m} \leq m-1$. If $\tilde{m}=m$ and $\tilde{q}<q$ then, because it is necessary that $f+1 \leq e$, one has that $\tilde{q}<q-1$. It follows that, if $\tilde{m}+\tilde{q}<m+q$, then $\tilde{m}+\tilde{q} \leq m+q-2$ and therefore $f+2 \leq e$. Because $(e+1) m-e \leq m+q-e$ and $\tilde{m}+\tilde{q}-f \leq$ $(f+2) \tilde{m}-f$, one has, because of (4.3), that (using also $\tilde{m} \leq m$ )

$$
(e+1) m-e \leq(f+2) m-f \text {. }
$$

But $f \leq e-2$, hence (4.4) becomes

$$
(e+1) m-e \leq e m-f,
$$

hence

$$
f \leq e-m \text {. }
$$

We are going to prove that

$$
f \leq e-\left(m^{t}-\sum\left(m^{i}: 1 \leq i \leq t-1\right)\right) \text { for each } t \in \mathbf{Z}_{\geq 1},
$$

which is, of course, impossible. It will follow that $\tilde{m}=m$ and $\tilde{q}=q$. We already proved it for $t=1$. Assume that it holds for some $t_{0} \in \mathbf{Z}_{\geq 1}$, hence

$$
f \leq e-\left(m^{t_{0}}-\sum\left(m^{i}: 1 \leq i \leq t_{0}-1\right)\right) .
$$

Hence, because of (4.4), we obtain that

$$
(e+1) m-e \leq\left[e-\left(m^{t_{0}}-\sum\left(m^{i}: 1 \leq i \leq t_{0}-1\right)\right)+2\right] m-f
$$

which gives us the desired inequality for $t_{0}+1$.

Now, assume that $\tau \in \mathbf{Z}_{\leq g+a}$ with $\tau \notin\left\{\alpha m+\beta q: \alpha, \beta \in \mathbf{Z}_{\geq 0}\right\}$ and assume that $\tau$ is a non-gap of $\bar{x}$. Then, because of (3), $\bar{x}$ would have at least $a+1$ non-gaps $c \leq g+a$. It would follow that $h^{0}((a+g) \bar{x}) \geq a$ +2 . In particular, because of the Semi-continuity Theorem, $h^{0}((a+g) x)$ $\geq a+2$. But, because the gap sequence of $x$ is the hyperordinary one, one has that $h^{0}((a+g) x)=a+1$. This gives a contradiction. Hence the gap sequence of $\bar{x}$ is the gap sequence described in the announcement of this theorem.

In order to understand the result of this theorem, I think it is necessary to give some examples of Weierstrass gap sequences which exist because of this theorem.

EXAMPLE 28. $n=3, m=5$.

For each $q \in \mathbf{Z}_{\geq 6}$, one has that (1) and (4) hold. 
Case $q=6$. Hence, $e=1$. Then (5) holds if $g<6$. Consider the case $g=5$ (which would give a Weierstrass point of maximal weight in this case). In this case $a=2$ and $\{\alpha 5+\beta 6 \leq 8\}=\{5,6\}$ consists of 2 elements, hence (3) holds. Hence $(1,2,3,4,7)$ is a Weierstrass gap sequence. Its weight is equal to 2 .

Case $q=7$. Hence, $e=1$. Then (5) holds if $g<7$. Let $g=6$. One proves that $(1,2,3,4,6,8)$ is a Weierstrass gap sequence. Its weight is equal to 3.

Case $q=8$. One proves that $(1,2,3,4,6,7)$ is a Weierstrass gap sequence.

And so on.

Finally, I give an example of a Weierstrass gap sequence of weight greater than its genus, whose existence follows from this theorem.

EXAMPLE 29. Let $n=10, m=15$ and $q=46$. One has that $e=3$ and $q \geq 40$ hence (1), (2) and (4) hold. Let $g=44$. Clearly (5) holds. Moreover $a=4$ and

$$
\{\alpha 15+\beta 46 \leq 49\}=\{15,30,45,46\}
$$

hence $(3)$ holds. Therefore $(1,2, \ldots, 14,16, \ldots, 29,31, \ldots, 44,47,48)$ is a Weierstrass gap sequence. Its genus is equal to 44 and its weight is equal to 50 .

\section{REFERENCES}

[1] E. Arbarello, Weierstrass points and moduli of curves, Compos. Math., 29 (1974), 325-342.

[2] E. Arbarello and M. Cornalba, Su una propreita' notevole dei morfismi di una curva a moduli generali in uno spazio proiettivo, Rend. Sem. Mat. Univers. Politecn. Torino, 38 (1980), 87-99.

[3] _ Su una congettura di Petri, Comment. Math. Helv., 56 (1981), 1-38.

[4] R. Buchweitz, On Zariski's criterion for equisingularity and non-smoothable monomial curves, preprint (1980).

[5] M. Coppens, A study of 4-gonal curves of genus $g \geq 7$, preprint No. 221, University of Utrecht (1981).

[6] _ Plane models of smooth curves over C, preprint No. 241, University of Utrecht (1982).

[7] _ One-dimensional linear systems of type II on smooth curves, Ph.-D. Thesis, Utrecht (1983).

[8] - The general curve which has a Weierstrass point which first non-gap is equal to $n$, preprint (1984).

[9] The Weierstrass gap sequences of the total ramification points of trigonal coverings of $\mathbf{P}^{1}$, to appear in Indag. Math. 
[10] S. Diaz, Tangent spaces in moduli via deformations with applications to Weierstrass points, to appear in Duke Math. J.

[11] Deformations of exceptional Weierstrass points, preprint.

[12] D. Eisenbud and J. Harris, Limit linear series, the irrationality of $\mathscr{M}_{g}$, and other applications, Bull. Amer. Math. Soc., 1 (1984), 277-280.

[13] A. Grothendieck, Technique de construction en géométrie analytique, Séminaire Cartan, $13(1960 / 61)$.

[14] R. C. Gunning, Lectures on Riemann surfaces, Princeton Math. Notes 2, Princeton U. Press (1970).

[15] R. Hartshorne, Algebraic Geometry, Graduate texts in Math. 52, Springer-Verlag, New York, Heidelberg Berlin (1977).

[16] K. Hensel and G. Landsberg, Theorie der algebraischen Funktionen einer Variabeln, Leibzig, Teubern (1902).

[17] E. Horikawa, On deformations of holomorphic maps I, J. Math. Soc. Japan, 25 (1973), 372-396.

[18] G. Kempf and D. Laksov, The determinental formula of Schubert calculus, Acta Math., 132 (1973), 153-162.

[19] J. Komeda, On Weierstrass points whose first non-gaps are four, J. Reine Angew. Math., 341 (1983), 68-86.

[20] H. Lange, Kurven mit rationaler Abbildungen, J. Reine Angew. Math., 295 (1977), 80-115.

[21] R. F. Lax, Weierstrass points on the universal curve, Math. Ann., 216 (1975), 35-42.

[22] R. F. Lax, Gap sequences and moduli in genus 4, Math. Z., 175 (1980), 67-75.

[23] C. Maclachlan, Weierstrass points on compact Riemann surfaces, J. London Math. Soc., 3 (1971), 722-724.

[24] D. Mumford, Abelian Varieties, Tata Inst. Studies in Math., Oxford University Press, London New York (1974).

[25] H. C. Pinkham, Deformations of algebraic varieties with $\mathscr{G}_{m}$ action, Astérisque, 20, Paris, Société Math. de France (1974).

[26] H. E. Rauch, Variational methods in the problem of the moduli of Riemann surfaces, Contribution to function theory, Bombay, Tata Institute of Fundamental research (1960).

[27] D. S. Rim and M. A. Vitulli, Weierstrass points and monomial curves, J. Algebra, 48 (1977), 454-476.

[28] A. Tannenbaum, Families of algebraic curves with nodes, Compos. Math., 41 (1980), 107-119.

[29] R. Waldi, Zur Konstruktion von Weierstrass Punkten mit vorgegebener Halbgruppe, Manuscripta Math., 30 (1980), 257-278.

Received April 24, 1986 and in revised form October 6, 1986.

Katholieke INDUSTRIEle HogeschoOl DER KeMPEN

Campus H.I.K. KleinhoefstraAt 4

B 2440-GeEL, Belgium 


\section{PACIFIC JOURNAL OF MATHEMATICS EDITORS}

\author{
V. S. VARADARAJAN \\ (Managing Editor) \\ University of California \\ Los Angeles, CA 90024 \\ Herbert Clemens \\ University of Utah \\ Salt Lake City, UT 84112 \\ R. FINN \\ Stanford University \\ Stanford, CA 94305
}

ROBION KIRBY

University of California

Berkeley, CA 94720

C. C. MOORE

University of California

Berkeley, CA 94720

HAROLD STARK

University of California, San Diego

La Jolla, CA 92093

\section{ASSOCIATE EDITORS}
R. ARENS
E. F. BECKENBACH
B. H. NEUMANN
F. WOLF
K. YOSHIDA (1906-1982)

\section{SUPPORTING INSTITUTIONS}
UNIVERSITY OF ARIZONA
UNIVERSITY OF OREGON
UNIVERSITY OF BRITISH COLUMBIA UNIVERSITY OF SOUTHERN CALIFORNIA
CALIFORNIA INSTITUTE OF TECHNOLOGY
UNIVERSITY OF CALIFORNIA
STANFORD UNIVERSITY
MONTANA STATE UNIVERSITY
UNIVERSITY OF HAWAII
UNIVERSITY OF NEVADA, RENO
UNIVERSITY OF TOKYO
NEW MEXICO STATE UNIVERSITY
UNIVERSITY OF UTAH
OREGON STATE UNIVERSITY
WASHINGTON STATE UNIVERSITY UNIVERSITY OF WASHINGTON 


\section{Pacific Journal of Mathematics}

\section{Vol. 131, No. $1 \quad$ November, 1988}

Tomek Bartoszynski, On covering of real line by null sets $\ldots \ldots \ldots \ldots \ldots 1$

Allen Davis Bell and Kenneth R. Goodearl, Uniform rank over differential operator rings and Poincaré-Birkhoff-Witt extensions $\ldots \ldots \ldots \ldots \ldots 13$

Brian Boe, Thomas Jones Enright and Brad Shelton, Determination of the intertwining operators for holomorphically induced representations of Hermitian symmetric pairs

Robert F. Brown, Topological identification of multiple solutions to

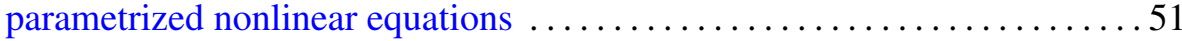

Marc R. M. Coppens, Weierstrass points with two prescribed nongaps ......71

Peter Larkin Duren and M. Schiffer, Grunsky inequalities for univalent functions with prescribed Hayman index .................... 105

Robert Greene and Hung-Hsi Wu, Lipschitz convergence of Riemannian manifolds

Kathryn E. Hare, Arithmetic properties of thin sets

Neal I. Koblitz, Primality of the number of points on an elliptic curve over a

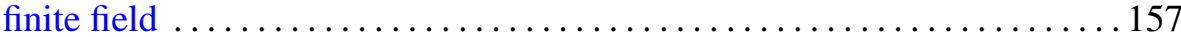

Isabel Dotti de Miatello and Roberto Jorge Miatello, Transitive isometry groups with noncompact isotropy

Raymond A Ryan, Weakly compact holomorphic mappings on Banach

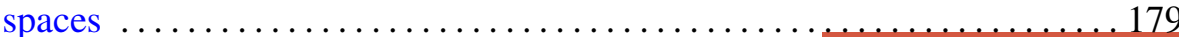

Tudor Zamfirescu, Curvature properties of typical convex surfaces 